\title{
Comments on new multiple-brane solutions based on Hata-Kojita duality in open string field theory
}

\author{
Toru Masuda \\ Institute of Physics, The University of Tokyo, \\ Komaba, Meguro-ku, Tokyo 153-8902, Japan \\ Department of Physics, Ochanomizu University, \\ Otsuka, Bunkyo-ku, Tokyo 2-1-1, Japan \\ E-mail: masudatoru@gmail.com
}

ABSTRACT: Recently, Hata and Kojita proposed a new energy formula for a class of solutions in Witten's open string field theory based on a novel symmetry of correlation functions they found. Their energy formula can be regarded as a generalization of the conventional energy formula by Murata and Schnabl. Following their proposal, we investigate their new ansatz for the classical solution representing double D-branes. We present a regularized definition of this solution and show that the solution satisfies the equation of motion when it is contracted with the solution itself and when it is contracted with any states in the Fock space. However, the Ellwood invariant and the boundary state of the solution are the same as those for the perturbative vacuum. This result disagrees with an expectation from the Ellwood conjecture.

Keywords: D-branes, Bosonic Strings, String Field Theory

ARXIV EPRINT: 1211.2649 


\section{Contents}

1 Introduction $\quad 2$

2 Review 4

2.1 The $K B c$ algebra 4

2.2 The inversion symmetry 5

2.3 Defining the solution as a limit 6

3 Regularization $\quad 7$

3.1 A class of $\delta$-sequence $\quad 7$

$\begin{array}{ll}3.2 & \text { Regularization of the identity state }\end{array}$

4 Definition of the double-brane solution $\quad 9$

$\begin{array}{ll}4.1 \text { Kinetic term } & 10\end{array}$

$\begin{array}{lll}4.2 & \text { Cubic term } & 12\end{array}$

4.3 Equation of motion 14

$\begin{array}{lll}4.4 & \text { The Ellwood invariant } & 15\end{array}$

$\begin{array}{lll}\text { 4.5 Boundary states } & 16\end{array}$

$\begin{array}{ll}\text { 4.6 Remarks on the ambiguity of classical solutions } & 16\end{array}$

$\begin{array}{lll}5 & \text { Summary } & 17\end{array}$

$\begin{array}{lr}\text { A Proof of (3.2) and (3.3) } & \mathbf{1 8}\end{array}$

$\begin{array}{ll}\text { B Some limits of correlation functions } & 18\end{array}$

C On the ansatz (4.2) for the double-brane solution $\quad \mathbf{2 0}$

C.1 Regularization 20

C.2 Regularized definition 21

$\begin{array}{lll}\text { C.3 Energy density } & 22\end{array}$

C.3.1 Kinetic term 22

C.3.2 Cubic term 23

C.4 Equation of motion contracted with states in the Fock space 24

$\begin{array}{ll}\text { D Notation } & \mathbf{2 6}\end{array}$

D.1 Conventions of the star-product 26

$\begin{array}{lll}\text { D.2 Definition of } K, B \text { and } c & 27\end{array}$

D.3 Correlation functions 28 


\section{Introduction}

Since the seminal study of Murata and Schnabl [1,2], solutions for multiple D-branes in Witten's open string field theory [3] have been intensively considered. Very recently, there has appeared an interesting paper by Hata and Kojita [4]. They proposed a new way to construct multiple-brane solutions. Their work can potentially reform our conventional understanding on this subject.

The starting point for the discussion is estimation of the energy density of the Okawatype solution,

$$
\Psi_{F}=F(K)^{2} c \frac{K B}{1-F(K)^{2}} c .
$$

Here $K, B$ and $c$ are symbols introduced to conveniently express a class of wedge states with operator insertions [5]. These three symbols satisfy simple algebraic relations called the $K B c$ subalgebra. We will review them in section 2.1. It is natural to expect that physical properties of the solution $\Psi_{F}$ are determined by choice of $F(K)$, which is a function of $K$. Murata and Schnabl derived a formula for the energy density of the solution (1.1) under some holomorphy conditions on $G(K)=1-F(K)^{2}$,

$$
\mathcal{E}\left(\Psi_{F}\right) \sim \frac{n_{0}}{2 \pi^{2} g_{o}^{2}}+(\text { anomalous term }) .
$$

Here $n_{a}$ denotes the order of the pole (or the multiplicity of the zero times minus one) of $G(z)$ at $z=a$. If we admit a formal object $1 / K^{n}$ and drop some surface terms, the anomalous term does not appear; however, it is not zero in the calculation of [2], except for the case $n_{0}= \pm 1,0$. Despite several efforts, fully acceptable definition of multiple-brane solutions based on (1.2) is not yet obtained. ${ }^{1}$

In [4], Hata and Kojita argued that the pole at $K=-\infty$ is in a sense equivalent to the pole at $K=0$, and it also contribute to the energy density. This argument arise from a novel symmetry of correlation functions in the $K B c$ subspace. This observation, together with (1.2), lead us to the following energy formula ${ }^{2}$

$$
\mathcal{E}\left(\Psi_{F}\right) \sim \frac{1}{2 \pi^{2} g_{o}^{2}}\left(n_{0}+n_{-\infty}\right)+(\text { anomalous term }) .
$$

From this formula, we can make new ansatzes for multiple-brane solutions.

We here note that some previous studies [6-10] do not appear to be consistent with (1.3). Still, it might be premature to dismiss (1.3). The pole at $K=-\infty$ is not fully considered so far, while it is related to the singularity of the identity string field. We empirically know that the identity string field requires quite careful treatment. Further investigation might resolve these apparent conflicts.

In this paper, we study the following ansatz for the classical solution presented in [4]: ${ }^{3}$

$$
\Psi=K c \frac{K}{1-K} B c .
$$

\footnotetext{
${ }^{1}$ In appendix $\mathrm{C}$, we summarize our attempt to construct the double-brane solution based on (1.2). Although it is not successful, the regularization method obtained there is essential in the present work.

${ }^{2}$ To be precise, the discussion of [4] is based on a particular regularization scheme, and the explicit form of the anomalous term is also derived.

${ }^{3}$ Notation used in [4] is different from ours. See appendix D for details.
} 
This expression is singular in the sense that the energy density of (1.4) is indefinite. We then define a double-brane solution as a limit of sequence of regular string fields as follows:

$$
\Psi=\lim _{\epsilon \rightarrow 0} K 1_{\epsilon} c \frac{K}{1-K} B c .
$$

Here $1_{\epsilon}$ is a regularized identity string field, defined by

$$
1_{\epsilon}=\int_{0}^{\infty} \delta_{\epsilon}(x) e^{x K}
$$

The $\delta$-sequence on the right-hand side of (1.6) has some special property, which is essential for our calculation. We will describe it in section 3.1. As an example of $\delta_{\epsilon}(x)$, we can take the following:

$$
\delta_{\epsilon}(x)=\frac{1}{\log \left(\epsilon^{-1}+1\right)} \frac{1}{(x+\epsilon)(x+\epsilon+1)} .
$$

We summarize properties of the solution (1.5) below:

(1) The solution satisfies the equation of motion when it is contracted with any state in the Fock space.

(2) The solution satisfies the equation of motion when it is contracted to the solution itself.

(3) The solution reproduces the energy density for double D-branes.

This is the first multiple-brane solution which satisfies both (1) and (2); however, it is still not clear whether (1) and (2) are sufficient to ensure that $\Psi$ is a solution to the equation of motion, since we do not know any good definition of the state space of the open string field theory. ${ }^{4}$ Indeed, as we will see in section 4.4 and section 4.5, some existing conjectures contradict with the interpretation that (1.5) is a classical solution representing double Dbranes. The property (3) is consistent with the formula (1.3). Since our calculation is completely independent of the argument of [4], this agreement is interesting.

We note that regularization of the multi-brane solution is also claimed in [4] ( $K_{\epsilon \eta}$ regularization). Yet, if one calculates the energy density of (1.4) under the $K_{\epsilon \eta}$ regularization, one needs to use an analytic continuation method called the $s-z$ trick [1] to obtain a finite value. Indeed, without the $s-z$ trick, one needs to drop singular terms by hand. This fact is explained in section 2.5 of [4]. This means that if one uses the $s$ - $z$ trick, then the result is not equal to the original expression in some cases. Since the $s$ - $z$ trick drastically simplifies calculation in many cases, and it is used in several studies, it is important to clarify that when it can be used as an identical transformation and when it cannot.

This paper is organized as follows: in section 2, we briefly introduce some preliminary materials and sketch a derivation of the formula (1.3). In section 3, we introduce the delta sequences appearing in (1.6), which is essential for our regularization. In section 4, we present the definition of the double-brane solution and check the equation of motion; and then we also calculate some physical quantities including the energy density, the Ellwood invariant and the boundary state. In section 5 , we summarize our results.

\footnotetext{
${ }^{4}$ In this paper, we use the word solution to refer to $\Psi$ in (1.5) for simplicity; however, it is not precise in this sense.
} 


\section{Review}

\subsection{The $K B c$ algebra}

In 2005, Schnabl constructed an analytic solution for tachyon condensation in Witten's open string field theory, using a class of wedge states with operator insertions [11]. The $K B c$ subalgebra was introduced by Okawa to express this class of wedge states with operator insertions [5]. Here $K$ is a grassman even object, and the wedge state $|n+1\rangle$ is represented as $e^{n K}$. The object $B$ is grassman odd, and it represents line integral of the anti-ghost. The object $c$ is also grassman odd, and it represents insertion of the $c$-ghost at the boundary. Together with the usual BRST operator $Q$, they satisfy the following algebraic relations:

$$
\begin{aligned}
& {[K, B]=0,} \\
& \{B, c\}=1 \text {, } \\
& c^{2}=B^{2}=0, \\
& Q K=0 \text {, } \\
& Q B=K, \\
& Q c=c K c \text {. }
\end{aligned}
$$

As described by Erler in [12], we can regard $K, B$ and $c$ as identity-based string fields. The commutation relations (2.1) then can be written using the star product: $[K, B]=K * B-B * K$, etc. ${ }^{5}$ The space of string fields which can be written by $K, B$ and $c$ is closed under the star multiplication and the action of $Q$. Thus we can use the $K B c$ subalgebra to find solutions to the equation of motion.

Consider the following formal solution to the equation of motion:

$$
\Psi_{F}=F(K)^{2} c \frac{K B}{1-F(K)^{2}} c .
$$

Here $F(K)$ is a function of $K$. Choice of $F(K)$ determines physical properties of the solution. For instance, the choice $F(K)=e^{K / 2}$ corresponds to Schnabl's original tachyon vacuum solution, while the choice $F(K)=(1-K)^{-1 / 2}$ corresponds to the simple tachyon vacuum solution by Erler and Schnabl [13]. Each of these two solutions reproduces the energy density of the tachyon vacuum. We can also construct solutions with zero energy density with a suitable choice of $F(K)$.

It is remarkable that the Okawa-type solution (2.3) can formally be written in the pure-gauge form:

$$
\Psi_{F}=U Q U^{-1}
$$

where

$$
U=1-F(K)^{2} B c, \quad \text { and } \quad U^{-1}=1+\frac{F(K)^{2}}{1-F(K)^{2}} B c .
$$

Since $U$ or $U^{-1}$ might be singular in general, $\Psi_{F}$ is not necessarily be a pure-gauge solution. For example, if we take $F(K)=(1-K)^{-1 / 2}$, which corresponds to the simple tachyon vacuum solution, then the string field $U$ has a factor $1 / K$. Generalizing the Okawa-type solution, a class of formal solutions which can formally be written as a pure-gauge form in the $K B c$ subalgebra is presented in [9].

We mostly follow the convention of [5], except for the overall factors of $K, B$ and $c$. See appendix D for details. For introduction to these topics including the $K B c$ subalgebra, see reviews $[14,15]$.

\footnotetext{
${ }^{5}$ The product symbol $*$ is usually omitted when we express string fields using $K, B$ and $c$.
} 


\subsection{The inversion symmetry}

In this subsection, we summarize a derivation of the formula (1.3). Let us start from the following homomorphisms of the $K B c$ subalgebra [10, 9],

$$
\hat{K}=f(K), \quad \hat{B}=\frac{f(K)}{K} B, \quad \hat{c}=c \frac{K}{f(K)} B c .
$$

These hatted objects, $\hat{K}, \hat{B}$ and $\hat{c}$, satisfy the same algebraic relations as the original $K, B$ and $c$ :

$$
\begin{aligned}
& {[\hat{K}, \hat{B}]=0,} \\
& \{\hat{B}, \hat{c}\}=1, \\
& \hat{c}^{2}=\hat{B}^{2}=0, \\
& Q \hat{K}=0 \text {, } \\
& Q \hat{B}=\hat{K}, \\
& Q \hat{c}=\hat{c} \hat{K} \hat{c} \text {. }
\end{aligned}
$$

Transformation law of the Okawa-type solution under these homomorphisms is simple,

$$
F(\hat{K})^{2} \hat{c} \frac{\hat{K} \hat{B}}{1-F(\hat{K})^{2}} \hat{c}=F(f(K))^{2} c \frac{K B}{1-F(f(K))^{2}} c .
$$

That is, $\hat{\Psi}_{F}=\Psi_{\hat{F}}$.

Let us concentrate on the special case $f(K)=1 / K$. We define $\tilde{K}, \tilde{B}$ and $\tilde{c}$ as

$$
\tilde{K}=\frac{1}{K}, \quad \tilde{B}=\frac{1}{K^{2}} B, \quad \tilde{c}=c K^{2} B c .
$$

Hata and Kojita proved the following symmetry of the correlation function (the inversion symmetry):

$$
\operatorname{tr}\left[\tilde{B} \tilde{c} e^{x_{1} \tilde{K}} \tilde{c} e^{x_{2} \tilde{K}} \tilde{c} e^{x_{3} \tilde{K}} \tilde{c} e^{x_{4} \tilde{K}}\right] \cong \operatorname{tr}\left[B c e^{x_{1} K} c e^{x_{2} K} c e^{x_{3} K} c e^{x_{4} K}\right]
$$

Note that in principle all the correlation functions in the $K B c$ subalgebra can be written using the four-point function on the right-hand side of (2.10); given a correlation function in the $K B c$ subalgebra, one can reduce the number of insertions of $B$ using the anti-commutation relation of $B$ and $c$. Insertions of $K$ s can be replaced with multiple differentiation of $x_{i}$. Thus, correlation functions in the $K B c$ subalgebra are invariant under the replacement of $(K, B, c)$ by $(\tilde{K}, \tilde{B}, \tilde{c})$ in general.

Now, suppose that we find the energy density $\mathcal{E}\left(\Psi_{F}\right)$ of the Okawa-type solution $\Psi_{F}$ for a choice of $F(K)$. If we replace $(K, B, c)$ in this calculation of $\mathcal{E}\left(\Psi_{F}\right)$ by $(\tilde{K}, \tilde{B}, \tilde{c})$, the resulting value does not change, because of the inversion symmetry (2.10); while the solution $\Psi_{F(K)}$ becomes $\Psi_{\tilde{F}(K)}=\Psi_{F(1 / K)}$, from (2.8), under this replacement. Therefore, the energy density of the solution $\Psi_{F}$ is the same as that of $\Psi_{F(1 / K)}$,

$$
\mathcal{E}\left(\Psi_{F(K)}\right)=\mathcal{E}\left(\Psi_{F(1 / K)}\right)
$$

We call this relation the Hata-Kojita duality. From (2.11) and (1.2), we are lead to (1.3). ${ }^{6}$

\footnotetext{
${ }^{6}$ Note that the pole of the function $G(K)=1-F(K)^{2}$ at $K=-\infty$ is not allowed in (1.2).
} 
Note that we used the symbol $\cong$ rather than $=$ in $(2.10)$. The reason is that the left-hand side has some singular terms, and the value is indefinite in the usual sense; if we define the string field $e^{x / K}$ as

$$
e^{x / K}=1-\int_{0}^{\infty} x_{0} F_{1}(; 2 ;-t x) e^{t K} d t
$$

where ${ }_{0} F_{1}(; a ; z)$ denotes a confluent hypergeometric function,

$$
{ }_{0} F_{1}(; a ; z)=\sum_{k=0}^{\infty} \frac{1}{(a)_{k}} \frac{z^{k}}{k !},
$$

then the left-hand side of (2.10) contains some identity-based terms, such as $\operatorname{tr}\left[B c K^{2} c K^{2} c^{2} K^{2}\right]$. To maintain the equivalence, we need to drop these terms. They are naturally dropped when we use the $s$ - $z$ trick.

\subsection{Defining the solution as a limit}

In this paper, we define the double-brane solution as a limit of a sequence of regular string fields (see (1.5)). That is, we consider one parameter family of string fields $\Psi_{\epsilon}$ with a small parameter $\epsilon>0$, and regard the limit $\Psi \equiv \lim _{\epsilon \rightarrow 0} \Psi_{\epsilon}$ as a solution to the equation of motion. We would like to clarify this point in the following.

For nonzero $\epsilon$, the string field $\Psi_{\epsilon}$ does not satisfy the equation of motion:

$$
\operatorname{eom}\left(\Psi_{\epsilon}\right) \equiv Q \Psi_{\epsilon}+\Psi_{\epsilon} * \Psi_{\epsilon} \neq 0
$$

We would like to require that the contraction of $\operatorname{eom}\left(\Psi_{\epsilon}\right)$ and any state $\varphi$ in the state space of the open string field theory vanishes as $\epsilon$ approaches 0 :

$$
\lim _{\epsilon \rightarrow 0+}\left\langle\varphi \mid \operatorname{eom}\left(\Psi_{\epsilon}\right)\right\rangle=0 .
$$

However, we do not know how to define the state space of the open string field theory. We then only require that $\lim _{\epsilon \rightarrow 0}\left\langle\varphi \mid \operatorname{eom}\left(\Psi_{\epsilon}\right)\right\rangle$ vanishes when $\varphi$ is any state in the Fock space and when $\varphi$ is the solution $\lim _{\epsilon \rightarrow 0} \Psi_{\epsilon}$ itself. Note that there is no relationship between these two requirements in general, for the state $\lim _{\epsilon \rightarrow 0} \Psi_{\epsilon}$ usually lies outside the Fock space.

When we calculate the physical quantities from the solution, we take the limit $\epsilon \rightarrow 0$ at the end of the calculation. For example, the energy density of $\Psi$ is defined as follows:

$$
\mathcal{E}(\Psi)=\lim _{\epsilon \rightarrow 0} \frac{1}{g_{o}^{2}}\left(\frac{1}{2} \operatorname{tr}\left[\Psi_{\epsilon} Q \Psi_{\epsilon}\right]+\frac{1}{3} \operatorname{tr}\left[\Psi_{\epsilon} \Psi_{\epsilon} \Psi_{\epsilon}\right]\right)
$$

We define the Ellwood invariant ${ }^{7}$ and the boundary state in a similar fashion. See section 4.4 and section 4.5 for details. Above treatment of the equation of motion and physical quantities reflect an expectation that the state space of the open string field theory is complete with respect to some norm.

\footnotetext{
${ }^{7}$ It is also commonly referred to as the gauge-invariant observable or the gauge-invariant overlap.
} 


\section{Regularization}

In this section, we describe the regularization method used in this paper. In section 3.1, we describe our delta sequence $\delta_{\epsilon}(x)$. In section 3.2 , we describe the regularized identity state $1_{\epsilon}$.

\subsection{A class of $\delta$-sequence}

Consider one parameter family of positive functions $\left\{\delta_{\epsilon}(x)\right\}$ with a small parameter $\epsilon>0$. We require the following conditions on $\delta_{\epsilon}(x)$ :

1. $\lim _{\epsilon \rightarrow 0} \int_{a}^{\infty} \delta_{\epsilon}(x) d x=0$ for ${ }^{\forall} a>0$.

2. $\int_{0}^{\infty} \delta_{\epsilon}(x) d x=1$ for ${ }^{\forall} \epsilon>0$.

Note that the lower limit of the integral in the condition 2 is zero. Since $\delta_{\epsilon}(x)$ is positive, $\int_{0}^{x} \delta_{\epsilon}(t) d t$ is a monotonically increasing function of $x$. Let $\lambda_{\epsilon}(t)$ be the inverse function of $\int_{0}^{x} \delta_{\epsilon}(t) d t$

$$
y=\int_{0}^{x} \delta_{\epsilon}(t) d t \quad \longleftrightarrow \quad x=\lambda_{\epsilon}(y) .
$$

We further require the following special condition on $\lambda_{\epsilon}(t)$ :

3. For $0<^{\forall} a<^{\forall} b<1$,

$$
\lim _{\epsilon \rightarrow 0+} \frac{\lambda_{\epsilon}(a)}{\lambda_{\epsilon}(b)}=0
$$

These three conditions characterize our delta sequence $\delta_{\epsilon}(t)$.

Now, we would like to prove the following property of $\delta_{\epsilon}(x)$ : if $f(s, t)$ is a bounded function on $(s, t) \in D$, where $D=[0, \infty) \times[0, \infty) \backslash\{(0,0)\}$, then it follows that ${ }^{8}$

$$
\lim _{\epsilon \rightarrow 0} \int_{0}^{\infty} d s \int_{0}^{\infty} d t \delta_{\epsilon}(s) \delta_{\epsilon}(t) f(s, t)=\frac{\lim _{a \rightarrow 0+} f(a, 0)+\lim _{a \rightarrow 0+} f(0, a)}{2} .
$$

To prove (3.1), we change the variables of integration on the left-hand side:

$$
\begin{aligned}
\int_{0}^{\infty} & d s_{1} \int_{0}^{\infty} d s_{2} \delta_{\epsilon}\left(s_{1}\right) \delta_{\epsilon}\left(s_{2}\right) f\left(s_{1}, s_{2}\right) \\
& =\int_{0}^{\infty} d s_{1} \int_{0}^{\infty} d s_{2} \frac{d h_{\epsilon}\left(s_{1}\right)}{d s_{1}} \frac{d h_{\epsilon}\left(s_{2}\right)}{d s_{2}} f\left(s_{1}, s_{2}\right) \\
& =\int_{0}^{1} d h_{1} \int_{0}^{1} d h_{2} f\left(\lambda_{\epsilon}\left(h_{1}\right), \lambda_{\epsilon}\left(h_{2}\right)\right) .
\end{aligned}
$$

Here, $h_{\epsilon}(s)$ in the second line denotes $h_{\epsilon}(s)=\int_{0}^{s} \delta_{\epsilon}(x) d x$. Let us divide the integration region of $\left(h_{1}, h_{2}\right)$ into three parts:

$$
T_{1} \equiv\left\{\left(h_{1}, h_{2}\right) \mid 0 \leq h_{1}<h_{2}<1\right\},
$$

\footnotetext{
${ }^{8}$ To be precise, we also assume that $f_{0}(x, y) \equiv \lim _{r \rightarrow 0+} f(x r, y r)((x, y) \in D)$ is continuous at $(1,0)$ and $(0,1)$.
} 


$$
\begin{aligned}
T_{2} & \equiv\left\{\left(h_{1}, h_{2}\right) \mid 0 \leq h_{2}<h_{1}<1\right\}, \\
F & \equiv\left\{\left(h_{1}, h_{2}\right) \mid 0 \leq h_{1}, h_{2} \leq 1,\left(h_{1}-h_{2}\right)\left(h_{1}-1\right)\left(h_{2}-1\right)=0\right\} .
\end{aligned}
$$

If $\left(h_{1}, h_{2}\right) \in T_{1}$, then the ratio $\lambda_{\epsilon}\left(h_{1}\right) / \lambda_{\epsilon}\left(h_{2}\right)$ converges to 0 as $\epsilon$ approaches 0 . From the conditions 1 and 2, we also see that both $\lambda_{\epsilon}\left(h_{1}\right)$ and $\lambda_{\epsilon}\left(h_{2}\right)$ converge to 0 as $\epsilon$ approaches 0 . Thus, we see the following:

$$
f\left(\lambda_{\epsilon}\left(h_{1}\right), \lambda_{\epsilon}\left(h_{2}\right)\right) \rightarrow \lim _{a \rightarrow 0} f(0, a), \quad\left(h_{1}, h_{2}\right) \in T_{1}
$$

We present a rigorous proof of (3.2) in appendix A. Similarly, assuming $\left(h_{1}, h_{2}\right) \in T_{2}$, the integrand $f\left(\lambda_{\epsilon}\left(h_{1}\right) \lambda_{\epsilon}\left(h_{2}\right)\right)$ converges to $\lim _{a \rightarrow 0} f(a, 0)$ as $\epsilon$ approaches 0 :

$$
f\left(\lambda_{\epsilon}\left(h_{1}\right), \lambda_{\epsilon}\left(h_{2}\right)\right) \rightarrow \lim _{a \rightarrow 0} f(a, 0), \quad\left(h_{1}, h_{2}\right) \in T_{2} .
$$

Since $f(s, t)$ is bounded, the integration over $F$ is zero. Therefore, we obtain (3.1). We can generalize (3.1) to multi-variable integrations. For three variables, we can prove that ${ }^{9}$

$$
\begin{gathered}
\lim _{\epsilon \rightarrow 0} \int_{0}^{\infty} d s_{1} \int_{0}^{\infty} d s_{2} \int_{0}^{\infty} d s_{3} \delta_{\epsilon}\left(s_{1}\right) \delta_{\epsilon}\left(s_{2}\right) \delta_{\epsilon}\left(s_{3}\right) g\left(s_{1}, s_{2}, s_{3}\right) \\
=\frac{1}{3} \lim _{a \rightarrow 0+}(g(a, 0,0)+g(0, a, 0)+g(0,0, a)) .
\end{gathered}
$$

As a simple example of $\delta_{\epsilon}(x)$, we may take

$$
\lambda_{\epsilon}(t)=\frac{1}{\epsilon^{\prime t-1}-1}-\frac{1}{\epsilon^{\prime-1}-1}, \quad \epsilon^{\prime}=\frac{\epsilon}{1+\epsilon},
$$

and

$$
\delta_{\epsilon}(x)=\frac{1}{\log \left(\epsilon^{-1}+1\right)} \frac{1}{(x+\epsilon)(x+\epsilon+1)} .
$$

Note that this choice satisfies the following stronger condition:

$3^{*}$. For $0<a<b<1$ and $0<r$,

$$
\lim _{\epsilon \rightarrow 0} \frac{\lambda_{\epsilon}(a)}{\lambda_{r \epsilon}(b)}=0
$$

Then, it follows that even if we change the ratio of two small parameters on the left-hand side of (3.1), the right-hand side does not change:

$$
\lim _{\epsilon \rightarrow 0} \int_{0}^{\infty} d s \int_{0}^{\infty} d t \delta_{\epsilon}(s) \delta_{r \epsilon}(t) f(s, t)=\frac{\lim _{a \rightarrow 0+} f(a, 0)+\lim _{a \rightarrow 0+} f(0, a)}{2}, \quad(0<r) .
$$

\footnotetext{
${ }^{9}$ We assume that $g(x, y, z)$ is a bounded function on $D^{(3)}=\{(x, y, z) \mid x, y, z \geq 0,(x, y, z) \neq(0,0,0)\}$, and $g_{0}(x, y, z) \equiv \lim _{r \rightarrow 0+} g(x r, y r, z r)$ is continuous at $(0,0,1),(0,1,0)$ and $(1,0,0)$.
} 


\subsection{Regularization of the identity state}

Using the delta sequence $\delta_{\epsilon}(x)$ in (3.5), we define the regularized identity state $1_{\epsilon}$,

$$
1_{\epsilon}=\int_{0}^{\infty} \delta_{\epsilon}(x) e^{x K}
$$

Some correlators in the $K B c$ subalgebra are singular, and their singularity is related to the identity string field. We can use the object $1_{\epsilon}$ to regularize some of these correlators. For example, we regularize the correlator $\operatorname{tr}[c K c K c K]$ as follows:

$$
\begin{aligned}
\lim _{\epsilon \rightarrow 0} \operatorname{tr} & {\left[c 1_{\epsilon} K c 1_{\epsilon} K c 1_{\epsilon} K\right] } \\
& =\lim _{a \rightarrow 0}\left(\lim _{b \rightarrow 0} \operatorname{tr}\left[c e^{a K} K c e^{b K} K c K\right]\right) \\
& =\lim _{x_{1} \rightarrow 0}\left(\lim _{x_{2} \rightarrow 0} \lim _{x_{3} \rightarrow 0} \frac{\partial}{\partial x_{1}} \frac{\partial}{\partial x_{2}} \frac{\partial}{\partial x_{3}} \operatorname{tr}\left[c e^{x_{1} K} c e^{x_{2} K} c e^{x_{3} K}\right]\right) \\
& =0 .
\end{aligned}
$$

where we used (3.4). An explicit form of the correlation function $\operatorname{tr}\left[c e^{x_{1} K} c e^{x_{2} K} c e^{x_{3} K}\right]$ is obtained by substituting $x_{4}=0$ in (D.2). Assuming (3.6), we can change the ratios of small parameters,

$$
\lim _{\epsilon \rightarrow 0} \operatorname{tr}\left[c 1_{\epsilon} K c 1_{\alpha \epsilon} K c 1_{\beta \epsilon} K\right]=0, \quad \alpha, \beta>0 .
$$

Taking the opportunity, we comment on the regularization of the identity-based solution in the $K B c$ subalgebra, $\Psi=-(1+K) c$. We define a regularized solution as follows:

$$
\Psi=-\lim _{\epsilon \rightarrow 0}(1+K) 1_{\epsilon} c .
$$

From (3.8), it follows that

$$
\operatorname{tr}[\Psi \Psi \Psi]=0
$$

Similarly, we find

$$
\operatorname{tr}[\Psi Q \Psi]=0 .
$$

That is, $\Psi$ satisfies the equation of motion when it is contracted with the solution itself, and its energy density is zero. This result agrees with (1.3). We can also show that $\Psi$ satisfies the equation of motion when it is contracted with any state in the Fock space. The energy density of $\Psi$ is the same as that of the perturbative vacuum, yet we are not sure whether it is a pure-gauge solution. In this case, the formula (2.4) does not give a regular $U$, for it contains a negative power of $K$. In terms of the winding number [16], this is a question whether winding numbers around $K=0$ are canceled by those of opposite sign around $K=-\infty$.

\section{Definition of the double-brane solution}

In this section, we give a definition of the double-brane solution. According to the energy formula (1.3), the following ansatz for the solution is expected to have the energy density of double D-branes:

$$
\Psi=K c \frac{K}{1-K} B c .
$$


The solution (4.1) is the symmetric counterpart of the following under the Hata-Kojita inversion:

$$
\Psi=\frac{1}{K} c \frac{K^{2} B}{K-1} c
$$

This is the familiar ansatz for the double-brane solution [1]. Since it contains the factor $1 / K$, the expression (4.2) itself is clearly singular. In contrast, the singularity of (4.1) is not so clear at first glance. However, there does exist an unobtrusive singularity, as essentially explained in [4], and the energy density of the solution is indefinite without suitable regularization. This seems to be consistent with the discussion by Erler [10], for the highest level in the dual $\mathcal{L}^{-}$level expansion of $\Psi$ is zero.

Now, let us present the regularized definition of the solution in question:

$$
\begin{aligned}
\Psi & =\lim _{\epsilon \rightarrow 0} K 1_{\epsilon} c \frac{K B}{1-K} c \\
& =\lim _{\epsilon \rightarrow 0} \int_{0}^{\infty} d x \delta_{\epsilon}(x)\left(\int_{0}^{\infty} d u e^{-u} \frac{\partial}{\partial x} \frac{\partial}{\partial u} e^{x K} c e^{u K} B c\right) .
\end{aligned}
$$

This solution reproduces the energy density of double D-branes. The equation of motion is satisfied when it is contracted with the solution itself and when it is contracted with any state in the Fock space. We also calculate the Ellwood invariant and the boundary state in section 4.4 and in section 4.5 , respectively. We will see that both of them are the same as those for the perturbative vacuum.

If we change the position where $1_{\epsilon}$ is inserted, then the properties of the solution drastically change. This means that we can make several distinct solutions with different properties from the ansatz (4.1). Note that this situation also occurs when we consider other ansatzes for solutions. We will discuss this subject in section 4.6.

\subsection{Kinetic term}

Let us calculate the normalized kinetic term $\widehat{\mathcal{E}}_{K}$ for the solution (4.3), defined by

$$
\widehat{\mathcal{E}}_{K}(\Psi)=\frac{\pi^{2}}{3}\langle\Psi, Q \Psi\rangle \text {. }
$$

Note that, if $\Psi$ is a multiple-brane solution, the quantity $\widehat{\mathcal{E}}_{K}+1$ represents the multiplicity of D-branes. Using the correlation function ${ }^{10}$

$$
C_{K}(x, y ; u, v) \equiv \operatorname{tr}\left[e^{x K} c e^{u K} B c Q\left(e^{y K} c e^{v K} B c\right)\right],
$$

we define the quantity $\mathcal{E}_{K}(\eta, \epsilon)$ as follows:

$$
\mathcal{E}_{K}(\epsilon, \eta)=\left.\int_{0}^{\infty} d u \int_{0}^{\infty} d v e^{-u-v}\left(\frac{\partial}{\partial x} \frac{\partial}{\partial y} \frac{\partial}{\partial u} \frac{\partial}{\partial v} C_{K}(x, y ; u, v)\right)\right|_{x=\epsilon, y=\eta} .
$$

Thanks to the relation (3.1), the regularized kinetic term can be expressed as ${ }^{11}$

$$
\widehat{\mathcal{E}}_{K}=\frac{\pi^{2}}{3} \frac{1}{2}\left(\lim _{\epsilon \rightarrow 0} \lim _{\eta \rightarrow 0}+\lim _{\eta \rightarrow 0} \lim _{\epsilon \rightarrow 0}\right) \mathcal{E}_{K}(\epsilon, \eta)=\frac{\pi^{2}}{3} \lim _{\epsilon \rightarrow 0} \lim _{\eta \rightarrow 0} \mathcal{E}_{K}(\epsilon, \eta) .
$$

\footnotetext{
${ }^{10}$ For an explicit form of this correlation function (4.4), see appendix D.3.

${ }^{11}$ To be precise, we need to prove that $\mathcal{E}_{K}(x, y)$ satisfies the conditions presented in footnote 8 before we use the relation (3.1). In appendix B, we check these conditions.
} 
By a straightforward calculation, we find

$$
\begin{aligned}
\mathcal{E}_{K}(\epsilon, \eta)= & \left.\int_{0}^{\infty} d u \int_{0}^{\infty} d v e^{-u-v}\left(\frac{\partial}{\partial x} \frac{\partial}{\partial y} \frac{\partial}{\partial u} \frac{\partial}{\partial v} C_{K}(x, y ; u, v)\right)\right|_{x=\epsilon, y=\eta} \\
= & \int_{0}^{\infty} d s \frac{4 e^{-s}}{(s+\eta+\epsilon)^{8}} \\
& \times\left(c(s, \epsilon, \eta) \cos \left(\frac{2 \pi \epsilon}{s+\epsilon+\eta}\right)+c(s, \eta, \epsilon) \cos \left(\frac{2 \pi \eta}{s+\epsilon+\eta}\right)\right. \\
& -\left((\epsilon+\eta) s^{6}+2(\epsilon+\eta)^{2} s^{5}+(\epsilon+\eta)^{3} s^{4}\right) \cos \left(\frac{2 \pi(\epsilon+\eta)}{s+\epsilon+\eta}\right) \\
& \left.+s(s, \epsilon, \eta) \sin \left(\frac{2 \pi \epsilon}{s+\epsilon+\eta}\right)+s(s, \eta, \epsilon) \sin \left(\frac{2 \pi \eta}{s+\epsilon+\eta}\right)\right) .
\end{aligned}
$$

Here we changed integration variables from $(u, v)$ to $(s, v) \equiv(u+v, v)$. The functions $c(s, \epsilon, \eta)$ and $s(s, \epsilon, \eta)$ are given by

$$
\begin{aligned}
c(s, \epsilon, \eta)=-\epsilon & s^{6}+2(\epsilon+\eta) s^{5}+\left(\epsilon^{2}+5 \epsilon \eta-2 \eta^{2}\right) s^{4}+2 \eta\left(\left(4+\pi^{2}\right) \epsilon^{2}-4 \eta^{2}\right) s^{3} \\
& +\eta\left(7 \epsilon^{3}+2\left(5+2 \pi^{2}\right) \epsilon^{2} \eta-10 \epsilon \eta^{2}-7 \eta^{3}\right) s^{2} \\
& +2 \eta\left(\epsilon^{4}+5 \epsilon^{3} \eta+\pi^{2} \epsilon^{2} \eta^{2}-5 \epsilon \eta^{3}-\eta^{4}\right) s \\
& \left.+3 \epsilon(\epsilon-\eta) \eta^{2}(\epsilon+\eta)^{2}\right) \\
s(s, \epsilon, \eta)=\pi \epsilon^{2}( & -s^{5}-2(\epsilon-\eta) s^{4}-\left(\epsilon^{2}+2 \epsilon \eta-12 \eta^{2}\right) s^{3} \\
& +2 \eta\left(-2 \epsilon^{2}+2 \epsilon \eta+7 \eta^{2}\right) s^{2}-\eta^{2}\left(\epsilon^{2}-6 \epsilon \eta-5 \eta^{2}\right) s \\
& \left.+2 \epsilon \eta^{3}(\epsilon+\eta)\right) .
\end{aligned}
$$

For finite $\epsilon$, we can change the order of the $s$-integral and the limit $\eta \rightarrow 0$. We then obtain that

$$
\begin{aligned}
\lim _{\eta \rightarrow 0} \mathcal{E}_{K}(\epsilon, \eta) & =-4 \pi \epsilon^{2} \int_{0}^{\infty} d s \frac{s^{3}}{(s+\epsilon)^{6}} e^{-s} \sin \left(\frac{2 \pi s}{s+\epsilon}\right) \\
& =-4 \pi \epsilon^{2} \sum_{j=0}^{\infty} \frac{(-1)^{j}}{(2 j+1) !} \int_{0}^{\infty} d s \frac{s^{3}}{(s+\epsilon)^{6}} e^{-s}\left(\frac{2 \pi s}{s+\epsilon}\right)^{2 j+1} .
\end{aligned}
$$

Using the integration formula below,

$$
\int_{0}^{\infty} d s e^{-s} \frac{s^{3}}{(s+\epsilon)^{6}} \frac{s^{2 j+1}}{(s+\epsilon)^{2 j+1}} \sim \frac{1}{(2 j+5)(2 j+6)} \frac{1}{\epsilon^{2}}+O\left(\epsilon^{-1}\right),
$$

we find that

$$
\begin{aligned}
\lim _{\epsilon \rightarrow 0} \lim _{\eta \rightarrow 0} \mathcal{E}_{K}(\epsilon, \eta) & =-4 \pi \lim _{\epsilon \rightarrow 0} \epsilon^{2} \sum_{j=0}^{\infty} \frac{(-1)^{j}}{(2 j+1) !}(2 \pi)^{2 j+1} \times \frac{1}{(2 j+5)(2 j+6)} \frac{1}{\epsilon^{2}} \\
& =-4 \pi \times\left(-\frac{3}{4 \pi^{3}}\right) \\
& =\frac{3}{\pi^{2}} .
\end{aligned}
$$


Therefore, we conclude that

$$
\widehat{\mathcal{E}}_{K}=1
$$

\subsection{Cubic term}

Let us move on to the cubic term. Let us define the regularized cubic term $\widehat{\mathcal{E}}_{C}$ as

$$
\widehat{\mathcal{E}}_{C}(\Psi)=-\frac{\pi^{2}}{3}\langle\Psi, \Psi * \Psi\rangle
$$

Note that the equation of motion contracted with $\Psi$ itself is equivalent to the condition $\widehat{\mathcal{E}}_{C}(\Psi)=\widehat{\mathcal{E}}_{K}(\Psi)$. We also define the quantity $\mathcal{E}_{C}\left(\epsilon_{1}, \epsilon_{2}, \epsilon_{3}\right)$ as follows:

$$
\begin{aligned}
\mathcal{E}_{C}\left(\epsilon_{1}, \epsilon_{2}, \epsilon_{3}\right)= & \int_{0}^{\infty} d u \int_{0}^{\infty} d v \int_{0}^{\infty} d w e^{-u-v-w} \\
& \times\left.\left(\frac{\partial}{\partial x} \frac{\partial}{\partial y} \frac{\partial}{\partial z} \frac{\partial}{\partial u} \frac{\partial}{\partial v} \frac{\partial}{\partial w} C_{C}(x, y, z ; u, v, w)\right)\right|_{x=\epsilon_{1}, y=\epsilon_{2}, z=\epsilon_{3}} .
\end{aligned}
$$

Using (3.4), $\widehat{\mathcal{E}}_{C}$ is expressed as

$$
\begin{aligned}
\widehat{\mathcal{E}}_{C}(\Psi) & =\frac{\pi^{2}}{3} \frac{1}{3} \lim _{\epsilon \rightarrow 0}\left(\mathcal{E}_{C}(\epsilon, 0,0)+\mathcal{E}_{C}(0, \epsilon, 0)+\mathcal{E}_{C}(0,0, \epsilon)\right) \\
& =\frac{\pi^{2}}{3} \lim _{\epsilon \rightarrow 0} \mathcal{E}_{C}(\epsilon, 0,0) .
\end{aligned}
$$

After a straightforward calculation, we find the following expression:

$$
\begin{aligned}
\mathcal{E}_{C} & \left(\epsilon_{1}, \epsilon_{2}, \epsilon_{3}\right) \\
= & \int_{0}^{\infty} d s \frac{e^{-s}}{\left(s+\epsilon_{1}+\epsilon_{2}+\epsilon_{3}\right)^{9}} \\
\times & \left(c_{1}\left(s, \epsilon_{1}, \epsilon_{2}, \epsilon_{3}\right) \cos \left(\frac{2 \pi \epsilon_{1}}{s+\epsilon_{1}+\epsilon_{2}+\epsilon_{3}}\right)+c_{1}\left(s, \epsilon_{2}, \epsilon_{3}, \epsilon_{1}\right) \cos \left(\frac{2 \pi \epsilon_{2}}{s+\epsilon_{1}+\epsilon_{2}+\epsilon_{3}}\right)\right. \\
& +c_{1}\left(s, \epsilon_{3}, \epsilon_{1}, \epsilon_{2}\right) \cos \left(\frac{2 \pi \epsilon_{3}}{s+\epsilon_{1}+\epsilon_{2}+\epsilon_{3}}\right)+c_{2}\left(s, \epsilon_{1}, \epsilon_{2}, \epsilon_{3}\right) \cos \left(\frac{2 \pi\left(\epsilon_{1}+\epsilon_{2}\right)}{s+\epsilon_{1}+\epsilon_{2}+\epsilon_{3}}\right) \\
& +c_{2}\left(s, \epsilon_{2}, \epsilon_{3}, \epsilon_{1}\right) \cos \left(\frac{2 \pi\left(\epsilon_{2}+\epsilon_{3}\right)}{s+\epsilon_{1}+\epsilon_{2}+\epsilon_{3}}\right)+c_{2}\left(s, \epsilon_{3}, \epsilon_{1}, \epsilon_{2}\right) \cos \left(\frac{2 \pi\left(\epsilon_{3}+\epsilon_{1}\right)}{s+\epsilon_{1}+\epsilon_{2}+\epsilon_{3}}\right) \\
& +c_{3}\left(s, \epsilon_{1}, \epsilon_{2}, \epsilon_{3}\right) \cos \left(\frac{2 \pi\left(\epsilon_{1}+\epsilon_{2}+\epsilon_{3}\right)}{s+\epsilon_{1}+\epsilon_{2}+\epsilon_{3}}\right)+s_{1}\left(s, \epsilon_{1}, \epsilon_{2}, \epsilon_{3}\right) \sin \left(\frac{2 \pi \epsilon_{1}}{s+\epsilon_{1}+\epsilon_{2}+\epsilon_{3}}\right) \\
& +s_{1}\left(s, \epsilon_{2}, \epsilon_{3}, \epsilon_{1}\right) \sin \left(\frac{2 \pi \epsilon_{2}}{s+\epsilon_{1}+\epsilon_{2}+\epsilon_{3}}\right)+s_{1}\left(s, \epsilon_{3}, \epsilon_{1}, \epsilon_{2}\right) \sin \left(\frac{2 \pi\left(\epsilon_{1}+\epsilon_{2}\right)}{s+\epsilon_{2}+\epsilon_{3}}\right)+s_{2}\left(s, \epsilon_{2}, \epsilon_{3}, \epsilon_{1}\right) \sin \left(\frac{2 \pi\left(\epsilon_{2}+\epsilon_{3}\right)}{s+\epsilon_{1}+\epsilon_{2}+\epsilon_{3}}\right) \\
& +s_{2}\left(s, \epsilon_{1}, \epsilon_{2}, \epsilon_{3}\right) \sin \left(\frac{2 \pi\left(\epsilon_{1}+\epsilon_{2}+\epsilon_{3}\right.}{s+\epsilon_{2}}\right) \\
& +s_{2}\left(s, \epsilon_{3}, \epsilon_{1}, \epsilon_{2}\right) \sin \left(\frac{2 \pi\left(\epsilon_{3}+\epsilon_{1}\right)}{s+\epsilon_{1}+\epsilon_{2}+\epsilon_{3}}\right)+s_{3}\left(s, \epsilon_{1}, \epsilon_{2}, \epsilon_{3}\right) \sin \left(\frac{2 \pi\left(\epsilon_{1}+\epsilon_{3}\right)}{s+\epsilon_{1}+\epsilon_{2}+\epsilon_{3}}\right)
\end{aligned}
$$


where

$$
\begin{aligned}
& c_{1}(s, x, y, z) \\
& =4 x^{2}\left(3 s^{6}+6 s^{5} x+9 s^{5} y+9 s^{5} z+3 s^{4} x^{2}+17 s^{4} x y+17 s^{4} x z+6 s^{4} y^{2}+12 s^{4} y z+6 s^{4} z^{2}\right. \\
& +2 \pi^{2} s^{3} x^{2} y+15 s^{3} x^{2} y+2 \pi^{2} s^{3} x^{2} z+15 s^{3} x^{2} z+12 s^{3} x y^{2}+24 s^{3} x y z+12 s^{3} x z^{2}-6 s^{3} y^{3} \\
& -18 s^{3} y^{2} z-18 s^{3} y z^{2}-6 s^{3} z^{3}+9 s^{2} x^{3} y+9 s^{2} x^{3} z+4 \pi^{2} s^{2} x^{2} y^{2}+18 s^{2} x^{2} y^{2}+8 \pi^{2} s^{2} x^{2} y z \\
& +36 s^{2} x^{2} y z+4 \pi^{2} s^{2} x^{2} z^{2}+18 s^{2} x^{2} z^{2}-6 s^{2} x y^{3}-18 s^{2} x y^{2} z-18 s^{2} x y z^{2}-6 s^{2} x z^{3}-9 s^{2} y^{4} \\
& -36 s^{2} y^{3} z-54 s^{2} y^{2} z^{2}-36 s^{2} y z^{3}-9 s^{2} z^{4}+2 s x^{4} y+2 s x^{4} z+12 s x^{3} y^{2}+24 s x^{3} y z+12 s x^{3} z^{2} \\
& +2 \pi^{2} s x^{2} y^{3}+3 s x^{2} y^{3}+6 \pi^{2} s x^{2} y^{2} z+9 s x^{2} y^{2} z+6 \pi^{2} s x^{2} y z^{2}+9 s x^{2} y z^{2}+2 \pi^{2} s x^{2} z^{3}+3 s x^{2} z^{3} \\
& -10 s x y^{4}-40 s x y^{3} z-60 s x y^{2} z^{2}-40 s x y z^{3}-10 s x z^{4}-3 s y^{5}-15 s y^{4} z-30 s y^{3} z^{2} \\
& -30 s y^{2} z^{3}-15 s y z^{4}-3 s z^{5}+3 x^{4} y^{2}+6 x^{4} y z+3 x^{4} z^{2}+3 x^{3} y^{3}+9 x^{3} y^{2} z+9 x^{3} y z^{2}+3 x^{3} z^{3} \\
& -3 x^{2} y^{4}-12 x^{2} y^{3} z-18 x^{2} y^{2} z^{2}-12 x^{2} y z^{3}-3 x^{2} z^{4}-3 x y^{5}-15 x y^{4} z-30 x y^{3} z^{2}-30 x y^{2} z^{3} \\
& \left.-15 x y z^{4}-3 x z^{5}\right) \text {, } \\
& c_{2}(s, x, y, z) \\
& =4(s+z)(x+y)\left(s^{6}+2 s^{5} z-3 s^{4} x^{2}-6 s^{4} x y-3 s^{4} y^{2}-2 s^{4} z^{2}-2 s^{3} x^{3}-6 s^{3} x^{2} y+2 \pi^{2} s^{3} x^{2} z\right. \\
& -6 s^{3} x y^{2}+4 \pi^{2} s^{3} x y z-3 s^{3} x z^{2}-2 s^{3} y^{3}+2 \pi^{2} s^{3} y^{2} z-3 s^{3} y z^{2}-8 s^{3} z^{3}+2 s^{2} x^{3} z+6 s^{2} x^{2} y z \\
& +4 \pi^{2} s^{2} x^{2} z^{2}+6 s^{2} x^{2} z^{2}+6 s^{2} x y^{2} z+8 \pi^{2} s^{2} x y z^{2}+12 s^{2} x y z^{2}-9 s^{2} x z^{3}+2 s^{2} y^{3} z+4 \pi^{2} s^{2} y^{2} z^{2} \\
& +6 s^{2} y^{2} z^{2}-9 s^{2} y z^{3}-7 s^{2} z^{4}+7 s x^{3} z^{2}+21 s x^{2} y z^{2}+2 \pi^{2} s x^{2} z^{3}+21 s x y^{2} z^{2}+4 \pi^{2} s x y z^{3} \\
& -9 s x z^{4}+7 s y^{3} z^{2}+2 \pi^{2} s y^{2} z^{3}-9 s y z^{4}-2 s z^{5}+3 x^{4} z^{2}+12 x^{3} y z^{2}+3 x^{3} z^{3}+18 x^{2} y^{2} z^{2} \\
& \left.+9 x^{2} y z^{3}-3 x^{2} z^{4}+12 x y^{3} z^{2}+9 x y^{2} z^{3}-6 x y z^{4}-3 x z^{5}+3 y^{4} z^{2}+3 y^{3} z^{3}-3 y^{2} z^{4}-3 y z^{5}\right), \\
& c_{3}(s, x, y, z)=-4(s+x+y+z)^{2} s^{4}(2 s-x-y-z)(x+y+z) \text {, } \\
& s_{1}(s, x, y, z) \\
& =-4 \pi x^{3}(s+y+z)(-s-x-y-z)\left(2 s^{3}+s^{2} x-4 s^{2} y-4 s^{2} z+3 s x y+3 s x z-6 s y^{2}\right. \\
& \left.-12 s y z-6 s z^{2}-2 x y^{2}-4 x y z-2 x z^{2}\right), \\
& s_{2}(s, x, y, z) \\
& =4 \pi e^{-s}(s+y)^{2}(x+z)^{2}(-s-x-y-z)\left(s^{3}-4 s^{2} y+4 s x y-5 s y^{2}+4 s y z-2 x y^{2}-2 y^{2} z\right) \text {, } \\
& s_{3}(s, x, y, z)=4 \pi s^{5}(x+y+z)^{2}(s+x+y+z) \text {. }
\end{aligned}
$$

As far as we keep $\epsilon_{1}$ finite, we can take the limits $\epsilon_{2} \rightarrow 0$ and $\epsilon_{3} \rightarrow 0$ before we perform the $s$ integral:

$$
\lim _{\epsilon_{2} \rightarrow 0} \lim _{\epsilon_{3} \rightarrow 0} \mathcal{E}_{C}\left(\epsilon_{1}, \epsilon_{2}, \epsilon_{3}\right)=-4 \pi \epsilon_{1}^{2} \int_{0}^{\infty} d s \frac{s^{3}}{\left(s+\epsilon_{1}\right)^{6}} e^{-s} \sin \left(\frac{2 \pi \epsilon_{1}}{s+\epsilon_{1}}\right) .
$$

This integral is the same as that appearing in (4.7). Therefore, we obtain

$$
\widehat{\mathcal{E}}_{C}=1 \text {. }
$$

From (4.8) and (4.10), we conclude that the energy density of the solution (4.3) is that of the double D-brane. 


\subsection{Equation of motion}

So far we have confirmed that the solution reproduces the energy density for double Dbranes. From (4.8) and (4.10), we can also conclude that the equation of motion is satisfied when it is contracted with the solution itself,

$$
\operatorname{tr}[\Psi Q \Psi]=-\operatorname{tr}[\Psi \Psi \Psi] \quad\left(=\frac{3}{\pi^{2}}\right) .
$$

Now, let us investigate the equation of motion contracted with states in the Fock space. It is apparently satisfied, for states in the Fock space always can be written as a wedge state of width one with local operator insertions.

Let $\phi$ be a state in the Fock space. Each term of the equation of motion can be written as follows:

$$
\begin{aligned}
\operatorname{tr}[\Psi \Psi \phi]= & \operatorname{tr}\left[K c \frac{K B}{1-K} c K c \frac{K B}{1-K} c \phi\right] \\
= & \int_{0}^{\infty} d u \int_{0}^{\infty} d v e^{-u-v} \frac{\partial}{\partial x} \frac{\partial}{\partial u} \frac{\partial}{\partial y} \frac{\partial}{\partial v} \operatorname{tr}\left[e^{x K} c e^{u K} B c e^{y K} c e^{v K} B c \phi\right] \\
= & \int_{0}^{\infty} d u \int_{0}^{\infty} d v e^{-u-v} \frac{\partial}{\partial x} \frac{\partial}{\partial u} \frac{\partial}{\partial y} \frac{\partial}{\partial v} \\
& \times\left(\operatorname{tr}\left[e^{x K} c e^{u K} e^{y K} c e^{v K} B c \phi\right]-\operatorname{tr}\left[e^{x K} c e^{u K} c e^{y K} e^{v K} B c \phi\right]\right) \\
= & \int_{0}^{\infty} d u \int_{0}^{\infty} d v e^{-u-v}\left(C_{\phi}^{(1,2,1)}(0, u, v)-C_{\phi}^{(1,1,2)}(0, u, v)\right), \\
\operatorname{tr}[(Q \Psi) \phi]= & \operatorname{tr}\left[\left(Q K c \frac{K B}{1-K} c\right) \phi\right] \\
= & \int_{0}^{\infty} d u e^{-u} \frac{\partial}{\partial x} \frac{\partial}{\partial u} \operatorname{tr}\left[\left(Q e^{x K} c e^{u K} B c\right) \phi\right] \\
= & \int_{0}^{\infty} d u e^{-u} \frac{\partial}{\partial x} \frac{\partial}{\partial u}\left(\operatorname{tr}\left[e^{x K} c K c e^{u K} B c \phi\right]-\operatorname{tr}\left[e^{x K} c e^{u K} c K B c \phi\right]\right) \\
= & \int_{0}^{\infty} d u e^{-u}\left(C_{\phi}^{(1,1,1)}(0,0, u)-C_{\phi}^{(1,1,1)}(0, u, 0)\right),
\end{aligned}
$$

where we defined

$$
C_{\phi}(x, y, z) \equiv \operatorname{tr}\left[e^{x K} c e^{y K} c e^{z K} B c \phi\right]
$$

and

$$
\left.C_{\phi}^{(i, j, k)}\left(x_{0}, y_{0}, z_{0}\right) \equiv \frac{\partial^{i}}{\partial x^{i}} \frac{\partial^{j}}{\partial y^{j}} \frac{\partial^{k}}{\partial z^{k}} C_{\phi}(x, y, z)\right|_{x=x_{0}, y=y_{0}, z=z_{0}} .
$$

Above expressions are valid as far as $C_{\phi}(x, y, z)$ is analytic around $(x, y, z)=(0, u, v)$, $(0,0, u)$ and $(0, u, 0)$. Since $C_{\phi}(x, y, z)$ is a correlation function of three local operator insertions with a line integral of $b$-ghost, $C_{\phi}(x, y, z)$ is regular for $0 \leq x+y+z<\infty$. Using integration by parts,

$$
\int_{0}^{\infty} d u e^{-u} C_{\phi}^{(1,2,1)}(0, u, v)=-C_{\phi}^{(1,1,1)}(0,0, v)+\int_{0}^{\infty} d u e^{-u} C_{\phi}^{(1,1,1)}(0, u, v),
$$


and

$$
\int_{0}^{\infty} d v e^{-v} C_{\phi}^{(1,1,2)}(0, u, v)=-C_{\phi}^{(1,1,1)}(0, u, 0)+\int_{0}^{\infty} d v e^{-v} C_{\phi}^{(1,1,1)}(0, u, v),
$$

we conclude that $\Psi$ satisfies the equation of motion contracted with any state $\phi$ in the Fock space:

$$
\operatorname{tr}[(Q \Psi) \phi]+\operatorname{tr}[\Psi \Psi \phi]=0 .
$$

\subsection{The Ellwood invariant}

In [6], Ellwood conjectured that there exists a relation between the gauge-invariant observables of open string field theory which were discovered in [17, 18], and the closed string tadpole on a disk. ${ }^{12}$ In this paper, we call these gauge-invariant observables the Ellwood invariant.

The Ellwood invariant for a classical solution $\Psi$ is defined by

$$
\mathcal{W}\left(\Psi, \phi_{\text {closed }}\right)=\left\langle\phi_{\text {closed }}(i) f_{I} \circ \Psi(0)\right\rangle_{\mathrm{UHP}} .
$$

Here, $\phi_{\text {closed }}$ is a closed string vertex operator of weight $(1,1)$ and ghost number $2 ; \Psi(0)$ is the operator corresponding to the classical solution $\Psi$, and $f_{I} \circ \Psi(0)$ is the conformal transformation of $\Psi(0)$ under the map associated with the identity state,

$$
f_{I}(\xi) \equiv \frac{2 \xi}{1-\xi^{2}}
$$

Ellwood conjectured that $\mathcal{W}\left(\Psi, \phi_{\text {closed }}\right)$ is equivalent to the difference of two tadpole diagrams,

$$
\mathcal{W}\left(\Psi, \phi_{\text {closed }}\right)=\mathcal{A}_{\Psi}\left(\phi_{\text {closed }}\right)-\mathcal{A}_{0}\left(\phi_{\text {closed }}\right)
$$

Here $\mathcal{A}_{0}\left(\phi_{\text {closed }}\right)$ denotes the closed string tadpole on a disk with the original boundary condition, and $\mathcal{A}_{\Psi}\left(\phi_{\text {closed }}\right)$ denotes the closed string tadpole with the boundary condition corresponding to the classical solution $\Psi$.

In $[1,2]$, Murata and Schnabl calculated the Ellwood invariant for the Okawa-type solution (1.1). Simply applying their formula to the solution (4.3), we find that the Ellwood invariant for the solution is zero,

$$
\mathcal{W}\left(\Psi, \phi_{\text {closed }}\right)=-\lim _{\epsilon \rightarrow 0}\left(\lim _{z \rightarrow 0} \frac{d F_{\epsilon}(z)^{2}}{d z} H_{\epsilon}(z)\right) \mathcal{A}_{0}\left(\phi_{\text {closed }}\right)=0
$$

where

$$
F_{\epsilon}(K)^{2}=\int_{0}^{\infty} d x \delta_{\epsilon}(x) e^{x K} K, \quad H_{\epsilon}(K)=\frac{K}{1-K} .
$$

This means that the Ellwood invariant of the solution $\Psi$ is that of the perturbative vacuum.

\footnotetext{
${ }^{12} \operatorname{In}[19,20]$, this conjecture was investigated in detail for the special case where $\phi_{\text {closed }}$ is a graviton. In particular, some correction to this relation was proposed in [20].
} 


\subsection{Boundary states}

In [7], Kiermaier, Okawa and Zwiebach constructed a closed string state $\left|B_{*}(\Psi)\right\rangle$ from classical solutions $\Psi$ of open string field theory. The closed string state is invariant under the gauge transformations of $\Psi$. For several known solutions, $\left|B_{*}(\Psi)\right\rangle$ corresponds to the boundary state of the vacuum which the classical solution $\Psi$ represents. We here simply refer to the closed string state $\left|B_{*}(\Psi)\right\rangle$ as the boundary state.

In $[8,9]$, the boundary states for different classical solutions in the $K B c$ subalgebra are calculated. Let $|B\rangle$ denote the boundary state for the perturbative vacuum. The boundary state for the Okawa-type solution (1.1) is given by

$$
\left|B_{*}\left(\Psi_{F}\right)\right\rangle=\frac{e^{(x+1) s}-e^{y s}}{e^{s}-1}|B\rangle
$$

where $^{13}$

$$
\begin{gathered}
x=\left.\frac{z}{1-F(z)^{2}}\left(\frac{1}{2} F(z)^{2}+2 F^{\prime}(z) F(z)\right)\right|_{z=0}, \\
y=\left.\frac{z}{1-F(z)^{2}}\left(\frac{1}{2} F(z)^{2}\right)\right|_{z=0} .
\end{gathered}
$$

From this formula, we find that the boundary state $\left|B_{*}(\Psi)\right\rangle$ for (4.3) is that of the perturbative vacuum,

$$
\left|B_{*}(\Psi)\right\rangle=|B\rangle .
$$

\subsection{Remarks on the ambiguity of classical solutions}

Now, let us slightly modify the definition of the solution (4.3). We consider the following solution:

$$
\Psi=\lim _{\epsilon \rightarrow 0} K c 1_{\epsilon} \frac{K B}{1-K} c .
$$

It is straightforward to calculate the energy or the Ellwood invariant of $\Psi$. We summarize properties of this solution as follows:

- The energy density of this solution is zero.

- The equation of motion is satisfied when it is contracted with the solution itself and when it is contracted with states in the Fock space.

- The Ellwood invariant is for the perturbative vacuum.

At least naively, both (4.11) and (4.3) can be considered as regularizations of (4.1). To define the solution without ambiguity, we need to regularize the solution and determine the order of limits. Note that these two solutions, (4.11) and (4.3), possess the same components at every level.

This kind of ambiguity is not limited to the ansatz (4.1). Take the identity-based solution $-(1+K) c$ for example. As stated in section 3.2, it can be regularized as (3.9).

\footnotetext{
${ }^{13}$ These expressions for $x$ and $y$ are valid only for the non-real solution (1.1). For more general expression, see [9].
} 
On the other hand, as described by Zeze in [21], we can also regularize it using the one parameter family of tachyon vacuum solutions that interpolates $-(1+K) c$ and the simple tachyon-vacuum solution as follows:

$$
\Psi=-\lim _{\epsilon \rightarrow 0} \frac{1-(\epsilon-1) K}{1-\epsilon K} c(1-\epsilon K) B c .
$$

Two regularized solutions, (3.9) and (4.12), are different in physical properties. The energy density of (3.9) is zero, while that of (4.12) is $-1 /\left(2 \pi^{2} g_{o}^{2}\right)$. It is hoped to gain a deeper understanding of different regularization methods and be able to predict the properties of the regularized solutions without calculating the physical quantities.

Let us here state one more question about the solution (4.3). The expression (4.1) can formally be written as a pure-gauge form as follows:

$$
\Psi=U Q U^{-1},
$$

where

$$
U=1-K B c, \quad U^{-1}=1+\frac{K}{1-K} B c .
$$

If we define the solution as (4.3), we expect that the solution is not true pure gauge. So, the gauge parameter $U$ or $U^{-1}$ must be singular in some sense. In particular, they must be disconnected to 1 . We need to understand in what sense it is singular and characterize the singularity.

\section{Summary}

We presented the double-brane solution (1.5) based on the ansatz of Hata and Kojita. The solution possesses finite energy density, which corresponds to the energy density of double D-branes. We also checked that the solution satisfies the equation of motion when it is contracted with the solution itself, and when it is contracted with any state of the Fock space. However, the Ellwood invariant and the boundary state are those for the perturbative vacuum. These inharmonious results make the physical interpretation of the solution difficult. Further research will be needed before the solution is fully accepted. In particular, we need to clarify the relation of our results and the discussion by Baba and Ishibashi [19], where the authors proved the correspondence between the energy density and the Ellwood invariant of classical solutions in part. It is also important to calculate the boundary state using the newly-proposed method by Kudrna, Maccaferri and Schnabl [22].

\section{Acknowledgments}

I would like to thank Yuji Okawa for valuable discussion and for detailed reading of the manuscript. I am indebted to Theodore Erler for valuable discussion and especially for important comments on my work, which helped me to correct some critical errors. I also would like to thank Toshiko Kojita, Toshifumi Noumi and Daisuke Takahashi for valuable and useful discussion. I also would like to thank Masaki Murata and Martin Schnabl for 
valuable discussion on the solution (4.2) and the anomaly of the equation of motion. I also would like to thank Hiroyuki Hata, Yuki Iimori, Mitsuhiro Kato, Shota Komatsu, Koichi Murakami and Shingo Torii for valuable discussion. I also thank Akiko Maruyama and Takayuki Yanagi for stimulating conversation. I acknowledge that the regularization method in section 3 and section B.1 is inspired by the formula for numerical integration of Iri, Moriguti and Takasawa [23]. Finally, I would like to thank the referee(s) for offering constructive suggestions leading to improvements of the manuscript.

\section{A Proof of (3.2) and (3.3)}

Let us prove (3.2) under the assumption presented in footnote 8. Using the $\epsilon-\delta$ definition of limit, the statement $f_{0}(x, y) \equiv \lim _{r \rightarrow 0} f(x r, y r)$ is expressed as

$$
{ }^{\forall} \varepsilon>0,{ }^{\exists} \delta_{1}(\varepsilon)>0 \text { such that } 0<r<\delta_{1}(\varepsilon) \rightarrow\left|f(x r, y r)-f_{0}(x, y)\right|<\varepsilon .
$$

Since $f_{0}(x, y)$ is continuous at $(x, y)=(0,1)$, it follows that

$$
{ }^{\forall} \varepsilon>0,{ }^{\exists} \delta_{2}(\varepsilon)>0 \text { such that } 0<r<\delta_{2}(\varepsilon) \rightarrow\left|f_{0}(r, 1)-f_{0}(0,1)\right|<\varepsilon .
$$

From the conditions 1, 2 and 3 in section 3.1, we also have

$$
{ }^{\forall} \varepsilon>0,{ }^{\exists} \delta_{3}(\varepsilon)>0 \text { such that } 0<r<\delta_{3}(\varepsilon) \rightarrow\left|\frac{\lambda_{r}\left(h_{1}\right)}{\lambda_{r}\left(h_{2}\right)}\right|<\varepsilon,
$$

and

$$
{ }^{\forall} \varepsilon>0,{ }^{\exists} \delta_{4}(\varepsilon)>0 \text { such that } 0<r<\delta_{4}(\varepsilon) \rightarrow\left|\lambda_{r}\left(h_{2}\right)\right|<\varepsilon .
$$

Now, setting $\delta_{5}(\epsilon) \equiv \min \left\{\delta_{4}\left(\delta_{1}(\varepsilon / 2)\right), \delta_{3}\left(\delta_{2}(\varepsilon / 2)\right)\right\}$, it follows that

$$
\begin{aligned}
0<r<\delta_{5}(\epsilon) \rightarrow & \left|f\left(\lambda_{r}\left(h_{1}\right), \lambda_{r}\left(h_{2}\right)\right)-f_{0}(0,1)\right| \\
& <\left|f\left(\frac{\lambda_{r}\left(h_{1}\right)}{\lambda_{r}\left(h_{2}\right)} \lambda_{r}\left(h_{2}\right), \lambda_{r}\left(h_{2}\right)\right)-f_{0}\left(\frac{\lambda_{r}\left(h_{1}\right)}{\lambda_{r}\left(h_{2}\right)}, 1\right)\right|+\left|f_{0}\left(\frac{\lambda_{r}\left(h_{1}\right)}{\lambda_{r}\left(h_{2}\right)}, 1\right)-f_{0}(0,1)\right| \\
< &
\end{aligned}
$$

Thus, noting $\lim _{a \rightarrow 0} f(0, a)=f_{0}(0,1)$, we obtain (3.2). In like manner, we can prove (3.3) and (3.4).

\section{B Some limits of correlation functions}

In this appendix, we explicitly calculate $\lim _{\epsilon \rightarrow 0} \mathcal{E}_{K}(a \epsilon, b \epsilon)$ and $\lim _{\epsilon \rightarrow 0} \mathcal{E}_{C}(a \epsilon, b \epsilon, c \epsilon)$. We start from the expression (4.6). We take up the first term and consider the following limit: for $0 \leq k \leq 6$,

$$
\begin{aligned}
\lim _{\epsilon \rightarrow 0} & \int_{0}^{\infty} d s \frac{s^{k}(a \epsilon)^{7-k}}{(s+a \epsilon+b \epsilon)^{8}} e^{-s} \cos \left(\frac{2 \pi a \epsilon}{s+a \epsilon+b \epsilon}\right) \\
& =\lim _{\epsilon \rightarrow 0} \int_{0}^{\infty} d s \frac{s^{k}}{(s+\alpha)^{8}} e^{-\epsilon s} \cos \left(\frac{2 \pi}{s+\alpha}\right) \\
& =\lim _{\epsilon \rightarrow 0} \sum_{l=0}^{\infty} \frac{(-)^{l}}{(2 l) !} \int_{0}^{\infty} d s \frac{s^{k}}{(s+\alpha)^{8}} e^{-\epsilon s}\left(\frac{2 \pi}{s+\alpha}\right)^{2 l}
\end{aligned}
$$


where we put $\alpha=1+b / a$. For $k=0$, the integral in this expression can be written as

$$
\int_{0}^{\infty} d s \frac{1}{(s+\alpha)^{8}} e^{-\epsilon s}\left(\frac{1}{s+\alpha}\right)^{2 l}=e^{\alpha \epsilon} \epsilon^{7+2 l} \Gamma(-7-2 l, \alpha \epsilon),
$$

where $\Gamma(z, \epsilon)$ denotes the incomplete gamma function defined by

$$
\Gamma(z, \epsilon) \equiv \int_{\epsilon}^{\infty} e^{-t} t^{z-1} d t .
$$

We now differentiate (B.2) with respet to $\epsilon$. Using the relations,

$$
\lim _{\epsilon \rightarrow 0} \epsilon^{k} \Gamma(-k, \epsilon)=\frac{1}{k} \quad(k \in \mathbb{N}),
$$

and

we obtain that

$$
\frac{d^{m}}{d \epsilon^{m}}\left[\epsilon^{k} \Gamma(-k, \epsilon)\right]=(-1)^{m} \epsilon^{k-m} \Gamma(-k+m, \epsilon),
$$

$$
\begin{aligned}
\lim _{\epsilon \rightarrow 0} \int_{0}^{\infty} d s \frac{s^{k}(a \epsilon)^{7-k}}{(s+a \epsilon+b \epsilon)^{8}} e^{-s} \cos \left(\frac{2 \pi a \epsilon}{s+a \epsilon+b \epsilon}\right) \\
\quad=(-1)^{k} \sum_{l=0}^{\infty} \frac{(2 \pi i)^{2 l}}{(2 l) !} \alpha^{k-2 l-7} \sum_{j=0}^{k}\left(\begin{array}{c}
k \\
j
\end{array}\right) \frac{(-1)^{j}}{7+2 l-j} \\
\quad=k ! \sum_{l=0}^{\infty} \frac{(2 \pi i)^{2 l}}{(2 l) !} \frac{(2 l+6-k) !}{(2 l+7) !} \alpha^{k-2 l-7}
\end{aligned}
$$

Note that this series can be expressed in terms of trigonometric functions.

Similarly, we can derive the following expressions: for $0 \leq k \leq 6$,

$$
\begin{aligned}
\lim _{\epsilon \rightarrow 0} \int_{0}^{\infty} d s \frac{s^{k}(a \epsilon)^{7-k}}{(s+a \epsilon+b \epsilon)^{8}} e^{-s} \sin \left(\frac{2 \pi a \epsilon}{s+a \epsilon+b \epsilon}\right) & =k ! \sum_{l=0}^{\infty} \frac{2 \pi(2 \pi i)^{2 l}}{(2 l+1) !} \frac{(2 l+7-k) !}{(2 l+8) !} \alpha^{k-2 l-8}, \\
\lim _{\epsilon \rightarrow 0} \int_{0}^{\infty} d s \frac{s^{k}(a \epsilon+b \epsilon)^{7-k}}{(s+a \epsilon+b \epsilon)^{8}} e^{-s} \cos \left(\frac{2 \pi(a \epsilon+b \epsilon)}{s+a \epsilon+b \epsilon}\right) & =\sum_{l=0}^{\infty} \frac{(2 \pi i)^{2 l}}{(2 l) !} \frac{(2 l+k) !(6-k) !}{(2 l+7) !} \\
\lim _{\epsilon \rightarrow 0} \int_{0}^{\infty} d s \frac{s^{k}(a \epsilon+b \epsilon)^{7-k}}{(s+a \epsilon+b \epsilon)^{8}} e^{-s} \sin \left(\frac{2 \pi(a \epsilon+b \epsilon)}{s+a \epsilon+b \epsilon}\right) & =\sum_{l=0}^{\infty} \frac{(2 \pi i)^{2 l+1} i}{(2 l+1) !} \frac{(2 l+k+1) !(6-k) !}{(2 l+8) !}
\end{aligned}
$$

Using above formulae, we obtain the following expressions:

$$
\begin{aligned}
\lim _{\epsilon \rightarrow 0} \mathcal{E}_{K}(a \epsilon, b \epsilon)= & \frac{2}{\pi^{2}}+\frac{(a+b)^{2}+2 \pi^{2} a b}{\pi^{2}(a+b)^{2}} \cos \left(\frac{2 a \pi}{a+b}\right)+\frac{a-b}{\pi(a+b)} \sin \left(\frac{2 a \pi}{a+b}\right), \\
\lim _{\epsilon \rightarrow 0} \mathcal{E}_{C}(a \epsilon, b \epsilon, c \epsilon)= & -\frac{3}{\pi^{2}}-\frac{(2 a-b-c)(a+b+c)^{2}+2 \pi^{2} a^{2}(b+c)}{\pi^{2}(a+b+c)^{3}} \cos \left(\frac{2 \pi a}{a+b+c}\right) \\
& -\frac{(2 b-c-a)(a+b+c)^{2}+2 \pi^{2} b^{2}(c+a)}{\pi^{2}(a+b+c)^{3}} \cos \left(\frac{2 \pi b}{a+b+c}\right) \\
& -\frac{(2 c-a-b)(a+b+c)^{2}+2 \pi^{2} c^{2}(a+b)}{\pi^{2}(a+b+c)^{3}} \cos \left(\frac{2 \pi c}{a+b+c}\right)
\end{aligned}
$$




$$
\begin{aligned}
& +\frac{3(a+b+c)^{2}-2 \pi^{2} a(a-2 b-2 c)}{2 \pi^{3}(a+b+c)^{2}} \sin \left(\frac{2 \pi a}{a+b+c}\right) \\
& +\frac{3(a+b+c)^{2}-2 \pi^{2} b(b-2 c-2 a)}{2 \pi^{3}(a+b+c)^{2}} \sin \left(\frac{2 \pi b}{a+b+c}\right) \\
& +\frac{3(a+b+c)^{2}-2 \pi^{2} c(c-2 a-2 b)}{2 \pi^{3}(a+b+c)^{2}} \sin \left(\frac{2 \pi c}{a+b+c}\right) .
\end{aligned}
$$

From these expressions, we see that $\mathcal{E}_{K}(x, y)$ satisfies the condition presented in footnote 8 , and $\mathcal{E}_{C}(x, y, z)$ satisfies the condition presented in footnote 9 , respectively.

\section{On the ansatz (4.2) for the double-brane solution}

Following Murata and Schnabl [1], several studies have been made to construct the multiple-brane solutions based on $(1.2)[2,16]$. The point here is that the expression $(4.2)$ contains a factor $1 / K$, and we need to regularize it. In this appendix, we summarize our attempt to construct the double-brane solution based on the ansatz (4.2). We show that the regularized solution satisfies the equation of motion when it is contracted with the solution itself. We also show that the equation of motion is broken when it is contracted with some states in the Fock space. These results are similar as those of $[2,16]$, where the solution is regularized using the $\epsilon$-regularization.

\section{C.1 Regularization}

Consider a string field $\varphi(\Lambda)$ with a large cutoff parameter $\Lambda$. We define a regularized string field $\varphi_{R}$ as follows:

$$
\varphi_{R} \equiv \lim _{\Lambda \rightarrow \infty} \varphi_{R}(\Lambda) \quad \text { with } \quad \varphi_{R}(\Lambda) \equiv \int_{0}^{1} d s \varphi(\lambda(\Lambda ; s)),
$$

where

$$
\lambda(\Lambda ; s) \equiv(\Lambda+1)^{s}-1, \quad 0 \leq s \leq 1 .
$$

The following property is important for our discussion:

$$
\lim _{\Lambda \rightarrow \infty} \frac{\lambda\left(\Lambda ; s_{1}\right)}{\lambda\left(\Lambda ; s_{2}\right)}= \begin{cases}\infty & s_{1}>s_{2}, \\ 0 & s_{2}>s_{1} .\end{cases}
$$

We now would like to prove an identity which is similar to (3.1). Let $\tilde{f}\left(\varphi\left(\Lambda_{1}\right), \varphi\left(\Lambda_{2}\right)\right)$ be a bilinear function of two $\varphi(\Lambda)$ s. For notational simplicity, we set $f\left(\Lambda_{1}, \Lambda_{2}\right) \equiv \tilde{f}\left(\varphi\left(\Lambda_{1}\right), \varphi\left(\Lambda_{2}\right)\right)$. We assume that the function $f\left(\Lambda_{1}, \Lambda_{2}\right)$ is bounded for $0 \leq \Lambda_{1}, \Lambda_{2}<\infty$. We also assume that the following limits exist:

$$
\lim _{a \rightarrow \infty}\left(\lim _{\Lambda \rightarrow \infty} f(\Lambda, a \Lambda)\right) \text { and } \lim _{a \rightarrow 0+}\left(\lim _{\Lambda \rightarrow \infty} f(\Lambda, a \Lambda)\right) .
$$

Under these conditions, we can prove the following identity:

$$
\lim _{\Lambda \rightarrow \infty} \tilde{f}\left(\varphi_{R}(\Lambda), \varphi_{R}(\Lambda)\right)=\frac{1}{2} \lim _{a \rightarrow \infty}\left(\lim _{\Lambda \rightarrow \infty} f(\Lambda, a \Lambda)\right)+\frac{1}{2} \lim _{a \rightarrow 0+}\left(\lim _{\Lambda \rightarrow \infty} f(\Lambda, a \Lambda)\right) .
$$


To prove (C.4), we divide the parameter space of $\left(s_{1}, s_{2}\right) \in[0,1] \times[0,1]$ into three parts:

$$
\begin{aligned}
& S_{1} \equiv\left\{\left(s_{1}, s_{2}\right) \mid 0 \leq s_{1}<s_{2} \leq 1\right\}, \\
& S_{2} \equiv\left\{\left(s_{1}, s_{2}\right) \mid 0 \leq s_{2}<s_{1} \leq 1\right\},
\end{aligned}
$$

and

$$
L \equiv\left\{\left(s_{1}, s_{2}\right) \mid 0 \leq s_{1}, s_{2} \leq 1, s_{1}=s_{2}\right\} .
$$

From (C.3), we see that if the parameters $\left(s_{1}, s_{2}\right)$ belong to $S_{1}$ or $S_{2}$, then the limit of the function $f\left(\lambda\left(\Lambda ; s_{1}\right), \lambda\left(\Lambda ; s_{2}\right)\right)$ as $\Lambda$ approaches $\infty$ can be expressed as follows:

$$
\begin{array}{ll}
\lim _{\Lambda \rightarrow \infty} f\left(\lambda\left(\Lambda ; s_{1}\right), \lambda\left(\Lambda ; s_{2}\right)\right)=\lim _{a \rightarrow \infty}\left(\lim _{\Lambda \rightarrow \infty} f(\Lambda, a \Lambda)\right), & \left(s_{1}, s_{2}\right) \in S_{1} \\
\lim _{\Lambda \rightarrow \infty} f\left(\lambda\left(\Lambda ; s_{1}\right), \lambda\left(\Lambda ; s_{2}\right)\right)=\lim _{a \rightarrow 0+}\left(\lim _{\Lambda \rightarrow \infty} f(\Lambda, a \Lambda)\right), & \left(s_{1}, s_{2}\right) \in S_{2} .
\end{array}
$$

Thus, we find that

$$
\begin{aligned}
\lim _{\Lambda \rightarrow \infty} & \tilde{f}\left(\varphi_{R}(\Lambda), \varphi_{R}(\Lambda)\right) \\
= & \lim _{\Lambda \rightarrow \infty} \int_{0}^{1} d s_{1} \int_{0}^{1} d s_{2} f\left(\lambda\left(\Lambda ; s_{1}\right), \lambda\left(\Lambda ; s_{2}\right)\right) \\
= & \lim _{\Lambda \rightarrow \infty} \iint_{S_{1}} d s_{1} d s_{2} f\left(\lambda\left(\Lambda ; s_{1}\right), \lambda\left(\Lambda ; s_{2}\right)\right)+\lim _{\Lambda \rightarrow \infty} \iint_{S_{2}} d s_{1} d s_{2} f\left(\lambda\left(\Lambda ; s_{1}\right), \lambda\left(\Lambda ; s_{2}\right)\right) \\
& +\lim _{\Lambda \rightarrow \infty} \iint_{L} d s_{1} d s_{2} f\left(\lambda\left(\Lambda ; s_{1}\right), \lambda\left(\Lambda ; s_{2}\right)\right) \\
= & \frac{1}{2} \lim _{a \rightarrow \infty}\left(\lim _{\Lambda \rightarrow \infty} f(\Lambda, a \Lambda)\right)+\frac{1}{2} \lim _{a \rightarrow 0+}\left(\lim _{\Lambda \rightarrow \infty} f(\Lambda, a \Lambda)\right) .
\end{aligned}
$$

In like manner, if $\tilde{f}_{3}$ is a bounded, trilinear function, we can show that

$$
\begin{aligned}
\lim _{\Lambda \rightarrow \infty} \tilde{f}_{3}\left(\varphi_{R}(\Lambda), \varphi_{R}(\Lambda), \varphi_{R}(\Lambda)\right)= & \frac{1}{3} \lim _{(a, b, c) \rightarrow(1,0,0)}\left(\lim _{\Lambda \rightarrow \infty} f_{3}(a \Lambda, b \Lambda, c \Lambda)\right) \\
& +\frac{1}{3} \lim _{(a, b, c) \rightarrow(0,1,0)}\left(\lim _{\Lambda \rightarrow \infty} f_{3}(a \Lambda, b \Lambda, c \Lambda)\right) \\
& +\frac{1}{3} \lim _{(a, b, c) \rightarrow(0,0,1)}\left(\lim _{\Lambda \rightarrow \infty} f_{3}(a \Lambda, b \Lambda, c \Lambda)\right) .
\end{aligned}
$$

Here $f_{3}\left(\Lambda_{1}, \Lambda_{2}, \Lambda_{3}\right)$ denotes $\tilde{f}\left(\varphi\left(\Lambda_{1}\right), \varphi\left(\Lambda_{2}\right), \varphi\left(\Lambda_{3}\right)\right)$, and we assumed that the limits on the right-hand side of (C.6) exist. To be precise, the identities (C.4) and (C.6) hold under milder conditions; however, we shall not pursue this matter here.

\section{C.2 Regularized definition}

The regularized form of the solution is given as follows:

$$
\Psi=-\lim _{\Lambda \rightarrow \infty} \int_{0}^{1} d s \int_{0}^{\lambda(\Lambda ; s)} d x e^{x K} c \frac{K^{2} B}{K-1} c .
$$


For convenience, we also define a string field $\Psi_{\text {cutoff }}(\Lambda)$ as

$$
\Psi_{\text {cutoff }}(\Lambda)=-\int_{0}^{\Lambda} d x e^{x K} c \frac{K^{2} B}{K-1} c
$$

Note that

$$
\Psi=\lim _{\Lambda \rightarrow \infty} \int_{0}^{1} d s \Psi_{\text {cutoff }}(\lambda(\Lambda ; s))
$$

which corresponds to the expression (C.1).

\section{C.3 Energy density}

In this subsection, we calculate the energy density of the solution (C.7).

\section{C.3.1 Kinetic term}

We start with evaluation of the normalized kinetic term $\widehat{\mathcal{E}}_{K}(\Psi)$ for the solution (C.7), defined by

$$
\widehat{\mathcal{E}}_{K}(\Psi)=\frac{\pi^{2}}{3}\langle\Psi, Q \Psi\rangle
$$

Using the correlation function $C_{K}(x, y ; u, v) \equiv \operatorname{tr}\left[e^{x K} c e^{u K} B c Q\left(e^{y K} c e^{v K} B c\right)\right]$, we define the quantity $\widehat{\mathcal{E}}_{K}\left(\Lambda_{1}, \Lambda_{2}\right)$ as follows:

$$
\begin{aligned}
\widehat{\mathcal{E}}_{K}\left(\Lambda_{1}, \Lambda_{2}\right) & =\frac{\pi^{2}}{3} \operatorname{tr}\left[\Psi_{\text {cutoff }}(\Lambda) Q \Psi_{\text {cutoff }}\left(\Lambda^{\prime}\right)\right] \\
& =\frac{\pi^{2}}{3} \int_{0}^{\infty} d u \int_{0}^{\infty} d v e^{-u-v} C_{K}^{(-1,-1,2,2)}\left(\Lambda, \Lambda^{\prime} ; u, v\right),
\end{aligned}
$$

where

$$
C_{K}^{(-1,-1,2,2)}(x, y ; u, v) \equiv \int_{0}^{x} d x^{\prime} \int_{0}^{y} d y^{\prime} \frac{\partial^{2}}{\partial u^{2}} \frac{\partial^{2}}{\partial v^{2}} C_{K}\left(x^{\prime}, y^{\prime} ; u, v\right) .
$$

From the relation (C.4), it follows that

$$
\widehat{\mathcal{E}}_{K}=\frac{1}{2} \lim _{a \rightarrow 0+}\left(\lim _{\Lambda \rightarrow \infty} \widehat{\mathcal{E}}_{K}(\Lambda, a \Lambda)\right)+\frac{1}{2} \lim _{a \rightarrow \infty}\left(\lim _{\Lambda \rightarrow \infty} \widehat{\mathcal{E}}_{K}(\Lambda, a \Lambda)\right) .
$$

We can carry out the differentiation with respect to $u$ and $v$ and the integration over $x^{\prime}$ and $y^{\prime}$ in (C.11) in a straightforward way. Since the integration over $u$ and $v$ in (C.10) is absolutely convergent, we can take the limit $\Lambda \rightarrow \infty$ before the integration. We then find that

$$
\lim _{\Lambda \rightarrow \infty} \widehat{\mathcal{E}}_{K}(\Lambda, a \Lambda)=\frac{1}{3}\left\{\left(1+\frac{2 a \pi^{2}}{(1+a)^{2}}\right) \cos \left(\frac{2 \pi}{1+a}\right)-\pi\left(1-\frac{2}{1+a}\right) \sin \left(\frac{2 \pi}{1+a}\right)+2\right\} .
$$

Plugging this expression into (C.12), we obtain that

$$
\widehat{\mathcal{E}}_{K}=1
$$




\section{C.3.2 Cubic term}

Let us move on to the cubic term. We define the regularized cubic term $\widehat{\mathcal{E}}_{C}$ as

$$
\widehat{\mathcal{E}}_{C}(\Psi)=-\frac{\pi^{2}}{3}\langle\Psi, \Psi * \Psi\rangle \text {. }
$$

We also define the quantity $\widehat{\mathcal{E}}_{C}\left(\Lambda_{1}, \Lambda_{2}, \Lambda_{3}\right)$ as follows:

$$
\begin{aligned}
& \widehat{\mathcal{E}}_{C}\left(\Lambda_{1}, \Lambda_{2}, \Lambda_{3}\right)= \operatorname{tr}\left[\Psi_{\text {cutoff }}\left(\Lambda_{1}\right) \Psi_{\text {cutoff }}\left(\Lambda_{2}\right) \Psi_{\text {cutoff }}\left(\Lambda_{3}\right)\right] \\
& \equiv \int_{0}^{\Lambda_{1}} d x \int_{0}^{\Lambda_{2}} d y \int_{0}^{\Lambda_{3}} d z \int_{0}^{\infty} d u \int_{0}^{\infty} d v \int_{0}^{\infty} d w \\
& \\
& \quad \times e^{-u-v-w} \frac{\partial^{2}}{\partial u^{2}} \frac{\partial^{2}}{\partial v^{2}} \frac{\partial^{2}}{\partial w^{2}} C_{C}(x, y, z ; u, v, w) .
\end{aligned}
$$

Then, using (C.6), $\widehat{\mathcal{E}}_{C}$ is given by

$$
\begin{aligned}
\widehat{\mathcal{E}}_{C}(\Psi)= & \frac{\pi^{2}}{3} \frac{1}{3} \lim _{(a, b, c) \rightarrow(1,0+, 0+)} \lim _{\Lambda \rightarrow \infty} \widehat{\mathcal{E}}_{C}(a \Lambda, b \Lambda, c \Lambda) \\
& +\frac{\pi^{2}}{3} \frac{1}{3} \lim _{(a, b, c) \rightarrow(0+, 1,0+)} \lim _{\Lambda \rightarrow \infty} \widehat{\mathcal{E}}_{C}(a \Lambda, b \Lambda, c \Lambda) \\
& +\frac{\pi^{2}}{3} \frac{1}{3} \lim _{(a, b, c) \rightarrow(0+, 0+, 1)} \lim _{\Lambda \rightarrow \infty} \widehat{\mathcal{E}}_{C}(a \Lambda, b \Lambda, c \Lambda) .
\end{aligned}
$$

It is straightforward to derive the following expression:

$$
\begin{aligned}
\lim _{\Lambda \rightarrow \infty} & \widehat{\mathcal{E}}_{C}(\Lambda, a \Lambda, b \Lambda) \\
= & 1+\frac{(1+a)^{2}+2 a \pi^{2}}{3(1+a)^{2}} \cos \left(\frac{2 \pi}{1+a}\right)+\frac{(1+b)^{2}+2 b \pi^{2}}{3(1+b)^{2}} \cos \left(\frac{2 \pi}{1+b}\right) \\
& +\frac{(a+b)^{2}+2 a b \pi^{2}}{3(a+b)^{2}} \cos \left(\frac{2 a \pi}{a+b}\right) \\
& +\frac{1}{3(1+a+b)^{3}}\left(\left\{-(1+a+b)^{2}(-1+2 a+2 b)-2(a+b)^{2} \pi^{2}\right\} \cos \left(\frac{2 \pi}{1+a+b}\right)\right. \\
& \quad+\left\{-(1+a+b)^{2}(2-a+2 b)-2 a(1+b)^{2} \pi^{2}\right\} \cos \left(\frac{2 a \pi}{1+a+b}\right) \\
& +\frac{(1-a) \pi}{3(1+a)} \sin \left(\frac{2 \pi}{1+a}\right)+\frac{(1-b) \pi}{3(1+b)} \sin \left(\frac{2 \pi}{1+b}\right) \\
& +\frac{(a-b) \pi}{3(a+b)} \sin \left(\frac{2 a \pi}{a+b}\right) \\
& +\frac{1}{6(1+a+b)^{2} \pi}\left(\left\{3(1+a+b)^{2}-2(-2+a+b)(a+b) \pi^{2}\right\} \sin \left(\frac{2 \pi}{1+a+b}\right)\right.
\end{aligned}
$$




$$
\begin{aligned}
& +\left\{3(1+a+b)^{2}-2(1+b-2 a)(1+b) \pi^{2}\right\} \sin \left(\frac{2 a \pi}{1+a+b}\right) \\
& \left.+\left\{3(1+a+b)^{2}-2(1+a-2 b)(1+a) \pi^{2}\right\} \sin \left(\frac{2 b \pi}{1+a+b}\right)\right) .
\end{aligned}
$$

From this expression, we find that

$$
\widehat{\mathcal{E}}_{C}=1
$$

\section{C.4 Equation of motion contracted with states in the Fock space}

From the calculation in the proceeding subsection, we conclude that the equation of motion is satisfied when it is contracted with the solution itself. Now, let us study the equation of motion contracted with states in the Fock space. For convenience, we define the string field $\Psi_{\Lambda}$ as follows:

$$
\Psi_{\Lambda} \equiv-\int_{0}^{1} d s \int_{0}^{\lambda(\Lambda ; s)} d x e^{x K} c \frac{K^{2} B}{K-1} c .
$$

The remainder of the equation of motion, $\operatorname{eom}\left(\Psi_{\Lambda}\right) \equiv \mathrm{Q} \Psi_{\Lambda}+\Psi_{\Lambda} * \Psi_{\Lambda}$, is not zero for finite $\Lambda$. Its explicit form is given by

$$
\operatorname{eom}\left(\Psi_{\Lambda}\right)=\frac{1}{K_{\Lambda}} c \frac{K}{K-1}\left(1-K \frac{1}{K_{\Lambda}}\right) c \frac{K^{2}}{K-1} B c-\frac{1}{K_{\Lambda}} c \frac{K}{K-1}\left(1-K \frac{1}{K_{\Lambda}}\right) c \frac{K^{2}}{K-1} B c
$$

where

$$
\frac{1}{K_{\Lambda}} \equiv-\int_{0}^{1} d s \int_{0}^{\lambda(\Lambda ; s)} d x e^{x K}
$$

We use the $\mathcal{L}_{0}$ Fock basis instead of the $L_{0}$ Fock basis. The $\mathcal{L}_{0}$ Fock basis is obtained by acting finite number of creation operators written in $z=(2 / \pi) \arctan \xi$ coordinates on the vacuum state $|0\rangle$. Let $\tilde{c}_{n}$ denotes oscillators of the $c$-ghost in the $z$ coordinates. For a few examples,

$$
c_{1}|0\rangle=\frac{\pi}{2} \tilde{c}_{1}|0\rangle
$$

and

$$
c_{0}|0\rangle=\left(\frac{\pi}{2}\right)^{2} \tilde{c}_{0}|0\rangle .
$$

The $\mathcal{L}_{0}$ level of the state $\phi$ is defined by its $\mathcal{L}_{0}$ eigenvalue plus one. We here calculate $\operatorname{tr}[\operatorname{eom}(\Psi(\Lambda)) \phi]$ for a few $\mathcal{L}_{0}$ levels. Let $\phi_{m, n}$ denotes a state of the form

$$
\phi_{n, m}=e^{K / 2} K^{n} c K^{m} e^{K / 2}
$$

After some calculation, we obtain

$$
\begin{aligned}
\operatorname{tr}\left[\operatorname{eom}(\Psi) \phi_{0,0}\right]=- & \frac{1}{2}\left(\lim _{a \rightarrow 0}+\lim _{a \rightarrow \infty}\right) \lim _{\Lambda \rightarrow \infty} \int_{0}^{\Lambda} d x \int_{0}^{\infty} d u \int_{0}^{\infty} d v e^{-u-v} \\
& \frac{\partial}{\partial u} \frac{\partial^{2}}{\partial v^{2}}\left\{F\left(\frac{1}{2}, \frac{1}{2}+x, v, a \Lambda+u\right)-F\left(\frac{1}{2}, \frac{1}{2}+x, a \Lambda+u, v\right)\right\}
\end{aligned}
$$




$$
\begin{aligned}
&=-\frac{1}{2}\left(\lim _{a \rightarrow 0}+\right.\left.\lim _{a \rightarrow \infty}\right)\left(\frac{1}{2 \pi^{2}}+\frac{1}{1+a}+\frac{-(1+a)^{2}+2 \pi^{2}}{2(1+a)^{2} \pi^{2}} \cos \left(\frac{2 a \pi}{1+a}\right)\right. \\
&+\frac{3+a}{2(1+a) \pi} \sin \left(\frac{2 a \pi}{1+a}\right)+\operatorname{Ci}(2 \pi)+\operatorname{Ci}\left(\frac{2 a \pi}{1+a}\right) \\
&\left.+\log \left(\frac{1+a}{a}\right)\right) \\
& \operatorname{tr}\left[\operatorname{eom}(\Psi) \phi_{1,0}\right]=-\left(\lim _{a \rightarrow 0}+\lim _{a \rightarrow \infty}\right) \lim _{\Lambda \rightarrow \infty} \int_{0}^{\Lambda} d x \int_{0}^{\infty} d u \int_{0}^{\infty} d v e^{-u-v} \\
&=-\frac{1}{2}(2-\gamma+\operatorname{Ci}(2 \pi)-\log (2 \pi)), \\
& \frac{\partial^{2}}{\partial v^{2}}\left\{F^{(1,0,0,0)}\left(\frac{1}{2}, \frac{1}{2}+x, v, a \Lambda+u\right)-F^{(1,0,0,0)}\left(\frac{1}{2}, \frac{1}{2}+x, a \Lambda+u, v\right)\right\} \\
&=-\left(\lim _{a \rightarrow 0}+\lim _{a \rightarrow \infty}\right)\left(\frac{1}{2 \pi^{2}}+\frac{1}{1+a}+\frac{-(1+a)^{2}+2 \pi^{2}}{2(1+a)^{2} \pi^{2}} \cos \left(\frac{2 a \pi}{1+a}\right)\right. \\
& \quad+\frac{3+a}{2(1+a) \pi} \sin \left(\frac{2 a \pi}{1+a}\right)+\mathrm{Ci}(2 \pi)+\operatorname{Ci}\left(\frac{2 a \pi}{1+a}\right) \\
&\left.\quad+\log \left(\frac{1+a}{a}\right)\right) \\
&=-2+\gamma-\operatorname{Ci}(2 \pi)+\log (2 \pi),
\end{aligned}
$$

and

$$
\operatorname{tr}\left[\operatorname{eom}(\Psi) \phi_{0,1}\right]=0 .
$$

Here $\mathrm{Ci}(x)$ denotes the cosine integral function

$$
\operatorname{Ci}(x)=-\int_{x}^{\infty} \frac{\cos t}{t} d t
$$

and $\gamma$ denotes the Euler-Mascheroni constant. The function $F\left(x_{1}, x_{2}, x_{3}, x_{4}\right)$ denotes the correlation function

$$
F\left(x_{1}, x_{2}, x_{3}, x_{4}\right)=\operatorname{tr}\left[B c e^{x_{1} K} c e^{x_{2} K} c e^{x_{3} K} c e^{x_{4} K}\right] .
$$

Its explicit form is presented in (D.2). We also used the notation

$$
F^{(1,0,0,0)}\left(x_{1}, x_{2}, x_{3}, x_{4}\right) \equiv \frac{\partial}{\partial x_{1}} F\left(x_{1}, x_{2}, x_{3}, x_{4}\right) .
$$

The correspondence between $\phi_{m, n}$ and the states in the $\mathcal{L}_{0}$ Fock space is given by

$$
\begin{aligned}
\tilde{c}_{1}|0\rangle & \sim e^{\frac{K}{2}} c e^{\frac{K}{2}}=\phi_{0,0}, \\
\mathcal{L}_{-1} \tilde{c}_{1}|0\rangle & \sim e^{\frac{K}{2}}(K c-c K) e^{\frac{K}{2}}=\phi_{1,0}-\phi_{0,1} .
\end{aligned}
$$

We also see that the following quantity is zero,

$$
\left\langle\operatorname{eom}(\Psi)\left|\mathcal{L}_{-1}^{\text {matter }} \tilde{c}_{1}\right| 0\right\rangle=0,
$$


since the matter one-point function vanishes. We then find that

$$
\begin{aligned}
& \left\langle\operatorname{eom}(\Psi)\left|\tilde{c}_{1}\right| 0\right\rangle=\frac{1}{2}(-2+\gamma-\mathrm{Ci}(2 \pi)+\log (2 \pi)) \quad(\sim 0.218827), \\
& \left\langle\operatorname{eom}(\Psi)\left|\tilde{c}_{0}\right| 0\right\rangle=\frac{1}{2}(-2+\gamma-\mathrm{Ci}(2 \pi)+\log (2 \pi)) .
\end{aligned}
$$

Thus, we conclude that the equation of motion is broken when it is contracted with some states in the Fock space. The constants presented in these expressions can be gathered into a single series,

$$
\gamma-\operatorname{Ci}(2 \pi)+\log (2 \pi)=-\frac{1}{2} \sum_{k=1}^{\infty} \frac{(-1)^{k}(2 \pi)^{2 k}}{k(2 k) !} .
$$

Comparison to the results in [2]. In [2], the remainder of the equation of motion under the $\epsilon$-regularization $[2,16]$ was minutely investigated. In our notation, the regularization of the ansatz (4.2) under the $\epsilon$-regularization is written as follows:

$$
\Psi_{\epsilon}=\frac{1}{K-\epsilon} c \frac{(K-\epsilon)^{2}}{K-\epsilon-1} B c .
$$

The remainder of the equation of motion $\operatorname{eom}\left(\Psi_{\epsilon}\right)$ is given by

$$
\operatorname{tr}\left[\operatorname{eom}\left(\Psi_{\epsilon}\right) \phi_{n, m}\right]=\operatorname{tr}\left[\frac{-\epsilon}{K-\epsilon} c \frac{(K-\epsilon)^{2}}{K-\epsilon-1} c \phi_{n, m}\right] .
$$

Using this expression, one can derive the following result:

$$
\begin{aligned}
\lim _{\epsilon \rightarrow 0}\left\langle\operatorname{eom}\left(\Psi_{\epsilon}\right)\left|\tilde{c}_{1}\right| 0\right\rangle \\
\quad=-\lim _{\epsilon \rightarrow 0} \int_{0}^{\infty} d x \int_{0}^{\infty} d y \epsilon e^{-\epsilon x-(1+\epsilon) y}\left(\frac{\partial}{\partial y}-\epsilon\right)^{2} \operatorname{tr}\left[e^{\left(x+\frac{1}{2}\right) K} c e^{y K} c e^{\frac{1}{2} K} c\right] \\
\quad=1
\end{aligned}
$$

This result is consistent with that obtained in [2] (see the paragraph including (3.15) in [2]).

From (C.27) and (C.31), we conclude that the remainder of the equation of motion depends on choice of the regularization.

\section{Notation}

In this appendix, we summarize our notation.

\section{D.1 Conventions of the star-product}

In this subsection, we clarify conventions of the star-product. ${ }^{14}$ Let $\Phi_{1}$ and $\Phi_{2}$ be open string fields. When we calculate the star-product $\Phi_{1} * \Phi_{2}$, we glue the right half

\footnotetext{
${ }^{14}$ To write this appendix, we consult the following textbook in part:

N. Ishibashi and K. Murakami, "String Field Theory — for a deeper understanding of string theory (Gen no ba no riron — gen riron no yori fukai rikai no tame ni)," Rinji Bessatsu Suuri Kagaku SGC Raiburari-92, Saiensu-sha, (2012) [ISSN0386-8257] (in Japanese).
} 
$(0 \leq \sigma \leq \pi / 2)$ of $\Phi_{1}$ to the left half $(\pi / 2 \leq \sigma \leq \pi)$ of $\Phi_{2}$. We call this convention the right-handed convention. This convention is convenient when we depict pictures of wedge states in the sliver coordinates.

On the other hand, in the original definition of the star-product [3], we glue the left half of $\Phi_{1}$ to the right half of $\Phi_{2}$ to calculate the star-product of $\Phi_{1}$ and $\Phi_{2}$. We call this convention the left-handed convention. In order to avoid possible confusion, we here write the star-product in the left-handed convention as $\left(\Phi_{1} * \Phi_{2}\right)_{L}$. Translation from one convention to the other is simple:

$$
\left(\Phi_{1} * \Phi_{2}\right)_{L}=(-1)^{\left|\Phi_{1}\right|\left|\Phi_{2}\right|} \Phi_{2} * \Phi_{1}
$$

Classical solutions in the left-handed convention $\Psi_{L}$ and that in the right-handed convention $\Psi$ are related as follows:

$$
\Psi_{L}=-\Psi
$$

Then, $\Psi_{L}$ and $\Psi$ satisfy the equation of motion as follows:

$$
\begin{aligned}
Q \Psi_{L}+\left(\Psi_{L} * \Psi_{L}\right)_{L} & =0, \\
Q \Psi+\Psi * \Psi & =0 .
\end{aligned}
$$

\section{D.2 Definition of $K, B$ and $c$}

The $K B c$ subalgebra is originally introduced to represent a class of wedge states with operator insertions. Let $|n+1\rangle$ denote the wedge state of width $n$. If $n$ is a natural number, then $|n+1\rangle$ can be written as follows:

$$
|n+1\rangle=\underbrace{|0\rangle * \cdots *|0\rangle}_{n} .
$$

Using $K$, it is expressed as follows:

$$
|n+1\rangle=e^{n K}
$$

All the functions of $K$ appearing in this paper are defined as a superposition of wedge states, except for the formal object $1 / K$ :

$$
f(K)=\int_{0}^{\infty} d x \widetilde{f}(x) e^{x K} .
$$

For example,

$$
\frac{1}{1-K}=\int_{0}^{\infty} d x e^{-x} e^{x K}
$$

and

$$
K=\left.\frac{\partial}{\partial x} e^{x K}\right|_{x \rightarrow 0+}\left(=\int_{0}^{\infty} d x \delta(x) \frac{\partial}{\partial x} e^{x K}\right) .
$$

This is a definition of $K$. 
To clarify the definition of $K, B$ and $c$, it is convenient to express them using the identity string field $|\mathcal{I}\rangle:{ }^{15}$

$$
\begin{aligned}
K & =\int_{i \infty}^{-i \infty} \frac{d z}{2 \pi i} T(z)|\mathcal{I}\rangle, \\
B & =\int_{i \infty}^{-i \infty} \frac{d z}{2 \pi i} B(z)|\mathcal{I}\rangle,
\end{aligned}
$$

and

$$
c=c(z=1)|\mathcal{I}\rangle .
$$

The coordinate system $z$ is defined by

$$
z=\frac{2}{\pi} \arctan \xi
$$

where $\xi$ represents the usual coordinate system on the upper half plane which is used in the radial quantization of the open string. Note that $K, B$ and $c$ are identity-based string fields, and some objects made from them are singular. For example, the value of $\operatorname{tr}[K c K c K c]$ is indefinite.

In the left-handed convention, definition of basic elements of the $K B c$ subalgebra is different from that in the right-handed convention:

$$
K_{L}=-K, \quad B_{L}=-B, \quad c_{L}=c .
$$

Under this definition, $K_{L}, B_{L}$ and $c_{L}$ satisfy the same algebraic relations as $K, B$ and $c$. As an example, let us write the solution (4.1) in the left-handed convention:

$$
\Psi=K c \frac{K}{1-K} B c \quad \Leftrightarrow \quad \Psi_{L}=c_{L} \frac{K_{L}}{1+K_{L}} B_{L} c_{L} K_{L} .
$$

At the end of this subsection, we clarify the overall factors of $K, B$ and $c$. Our $K$ and $B$ are $\pi / 2$ times those of Okawa's original definition [5], which we here write as $K_{\text {Okawa }}$ and $B_{\text {Okawa }}$, respectively; our $c$ is $2 / \pi$ times that of the original definition, which we write as $c_{\text {Okawa }}$ :

$$
K=\frac{\pi}{2} K_{\text {Okawa }}, \quad B=\frac{\pi}{2} B_{\text {Okawa }}, \quad c=\frac{2}{\pi} c_{\text {Okawa }} .
$$

\section{D.3 Correlation functions}

We use the notation $\operatorname{tr}[\ldots]$ to represent correlation functions in the $K B c$ subalgebra. In our notation, the four point function is expressed as follows:

$$
\begin{gathered}
\operatorname{tr}\left[B c e^{x_{1} K} c e^{x_{2} K} c e^{x_{3} K} c e^{x_{4} K}\right] \equiv\left\langle\mathcal{B}_{L} c(0) c\left(x_{1}\right) c\left(x_{1}+x_{2}\right) c\left(x_{1}+x_{2}+x_{3}\right)\right\rangle_{C_{x_{1}+x_{2}+x_{3}+x_{4}}} \\
=-\frac{s^{2}}{4 \pi^{3}}\left(x_{3} \sin \frac{2 \pi x_{1}}{s}-\left(x_{2}+x_{3}\right) \sin \frac{2 \pi\left(x_{1}+x_{2}\right)}{s}+x_{2} \sin \frac{2 \pi\left(x_{1}+x_{2}+x_{3}\right)}{s}\right. \\
\left.\quad+x_{1} \sin \frac{2 \pi x_{3}}{s}-\left(x_{1}+x_{2}\right) \sin \frac{2 \pi\left(x_{2}+x_{3}\right)}{s}+\left(x_{1}+x_{2}+x_{3}\right) \sin \frac{2 \pi x_{2}}{s}\right),
\end{gathered}
$$

\footnotetext{
${ }^{15}$ The identity string field $|\mathcal{I}\rangle$ is usually denoted by 1 when we express string fields using $K, B$ and $c$, as in (2.1).
} 
with

$$
s=x_{1}+x_{2}+x_{3}+x_{4} .
$$

Here $C_{r}$ denotes a semi-infinite cylinder of circumference $r$. The character $s$ represents the circumference of the cylinder; $\mathcal{B}_{L}$ denotes a line integral of the $b$-ghost $\int_{i \infty}^{-i \infty} d z b(z)$. In this case, the path of integration is along the line $\operatorname{Re}(z)=0-$. Note that (D.2) is not smooth at $\left(x_{1}, x_{2}, x_{3}, x_{4}\right)=(0,0,0,0)$. This is a source of the singularity of correlation functions discussed in section 3.2 .

The following two correlators are used when we calculate the energy density of classical solutions:

$$
\begin{aligned}
& C_{K}(x, y ; u, v) \equiv \operatorname{tr}\left[e^{x K} c e^{u K} B c Q\left(e^{y K} c e^{v K} B c\right)\right] \\
&= \frac{1}{2 \pi^{2}}\left\{-(x+y) s+y(s-x) \cos \frac{2 \pi x}{s}+x(s-y) \cos \frac{2 \pi y}{s}+u v \cos \frac{2 \pi u}{s}+u v \cos \frac{2 \pi v}{s}\right. \\
&\left.+(x y-u v) \cos \frac{2 \pi(x+v)}{s}+(x y-u v) \cos \frac{2 \pi(y+v)}{s}\right\} \\
&+\frac{s}{4 \pi^{3}}\left\{2 y \sin \frac{2 \pi x}{s}+2 x \sin \frac{2 \pi y}{s}+(s-2 v) \sin \frac{2 \pi u}{s}+(s-2 u) \sin \frac{2 \pi v}{s}\right. \\
&\left.+(x-y+u-v) \sin \frac{2 \pi(x+v)}{s}+(-x+y+u-v) \sin \frac{2 \pi(y+v)}{s}\right\}
\end{aligned}
$$

with

$$
s=x+y+u+v,
$$

and

$$
\begin{aligned}
C_{C}(x, y, z ; u, v, w) \equiv \operatorname{tr}\left[e^{x K} c e^{u K} B c e^{y K} c B e^{v K} c e^{z K} c B e^{w K} c\right] \\
=\frac{s^{2}}{4 \pi^{3}} x\left(\sin \frac{2 \pi v}{s}-\sin \frac{2 \pi(v+y)}{s}-\sin \frac{2 \pi(v+z)}{s}+\sin \frac{2 \pi(v+y+z)}{s}\right) \\
+\frac{s^{2}}{4 \pi^{3}} y\left(\sin \frac{2 \pi w}{s}-\sin \frac{2 \pi(w+z)}{s}-\sin \frac{2 \pi(w+x)}{s}+\sin \frac{2 \pi(w+z+x)}{s}\right) \\
+\frac{s^{2}}{4 \pi^{3}} z\left(\sin \frac{2 \pi u}{s}-\sin \frac{2 \pi(u+x)}{s}-\sin \frac{2 \pi(u+y)}{s}-\sin \frac{2 \pi(u+x+y)}{s}\right),
\end{aligned}
$$

with

$$
s=x+y+z+u+v+w .
$$

Open Access. This article is distributed under the terms of the Creative Commons Attribution License (CC-BY 4.0), which permits any use, distribution and reproduction in any medium, provided the original author(s) and source are credited.

\section{References}

[1] M. Murata and M. Schnabl, On multibrane solutions in open string field theory, Prog. Theor. Phys. Suppl. 188 (2011) 50 [arXiv:1103.1382] [INSPIRE].

[2] M. Murata and M. Schnabl, Multibrane solutions in open string field theory, JHEP 07 (2012) 063 [arXiv:1112.0591] [INSPIRE]. 
[3] E. Witten, Noncommutative geometry and string field theory, Nucl. Phys. B 268 (1986) 253 [INSPIRE].

[4] H. Hata and T. Kojita, Singularities in K-space and multi-brane solutions in cubic string field theory, JHEP 02 (2013) 065 [arXiv: 1209.4406] [INSPIRE].

[5] Y. Okawa, Comments on Schnabl's analytic solution for tachyon condensation in Witten's open string field theory, JHEP 04 (2006) 055 [hep-th/0603159] [INSPIRE].

[6] I. Ellwood, The closed string tadpole in open string field theory, JHEP 08 (2008) 063 [arXiv:0804.1131] [INSPIRE].

[7] M. Kiermaier, Y. Okawa and B. Zwiebach, The boundary state from open string fields, arXiv:0810.1737 [INSPIRE].

[8] D. Takahashi, The boundary state for a class of analytic solutions in open string field theory, JHEP 11 (2011) 054 [arXiv: 1110.1443] [INSPIRE].

[9] T. Masuda, T. Noumi and D. Takahashi, Constraints on a class of classical solutions in open string field theory, JHEP 10 (2012) 113 [arXiv: 1207.6220] [INSPIRE].

[10] T. Erler, The identity string field and the Sliver frame level expansion, JHEP 11 (2012) 150 [arXiv: 1208.6287] [INSPIRE].

[11] M. Schnabl, Analytic solution for tachyon condensation in open string field theory, Adv. Theor. Math. Phys. 10 (2006) 433 [hep-th/0511286] [InSPIRE].

[12] T. Erler, Split string formalism and the closed string vacuum, JHEP 05 (2007) 083 [hep-th/0611200] [INSPIRE].

[13] T. Erler and M. Schnabl, A simple analytic solution for tachyon condensation, JHEP 10 (2009) 066 [arXiv:0906.0979] [INSPIRE].

[14] M. Schnabl, Algebraic solutions in open string field theory - a lightning review, Acta Polytechnica 50 (2010) 102 [arXiv:1004.4858] [INSPIRE].

[15] Y. Okawa, Analytic methods in open string field theory, Prog. Theor. Phys. 128 (2012) 1001 [INSPIRE].

[16] H. Hata and T. Kojita, Winding number in string field theory, JHEP 01 (2012) 088 [arXiv:1111.2389] [INSPIRE].

[17] A. Hashimoto and N. Itzhaki, Observables of string field theory, JHEP 01 (2002) 028 [hep-th/0111092] [INSPIRE].

[18] D. Gaiotto, L. Rastelli, A. Sen and B. Zwiebach, Ghost structure and closed strings in vacuum string field theory, Adv. Theor. Math. Phys. 6 (2003) 403 [hep-th/0111129] [INSPIRE].

[19] T. Baba and N. Ishibashi, Energy from the gauge invariant observables, JHEP 04 (2013) 050 [arXiv: 1208.6206] [INSPIRE].

[20] H. Hata and T. Kojita, Inversion symmetry of gravitational coupling in cubic string field theory, JHEP 12 (2013) 019 [arXiv:1307.6636] [INSPIRE].

[21] S. Zeze, Regularization of identity based solution in string field theory, JHEP 10 (2010) 070 [arXiv: 1008.1104] [INSPIRE].

[22] M. Kudrna, C. Maccaferri and M. Schnabl, Boundary state from Ellwood invariants, JHEP 07 (2013) 033 [arXiv: 1207.4785] [INSPIRE].

[23] M. Iri, S. Moriguti and Y. Takasawa, On a certain quadrature formula (in Japanese), Kokyuroku Res. Inst. Math. Sci. Kyoto Univ. 91 (1970) 82 [English translation in J. Comp. Appl. Math. 17 (1987) 3]. 